

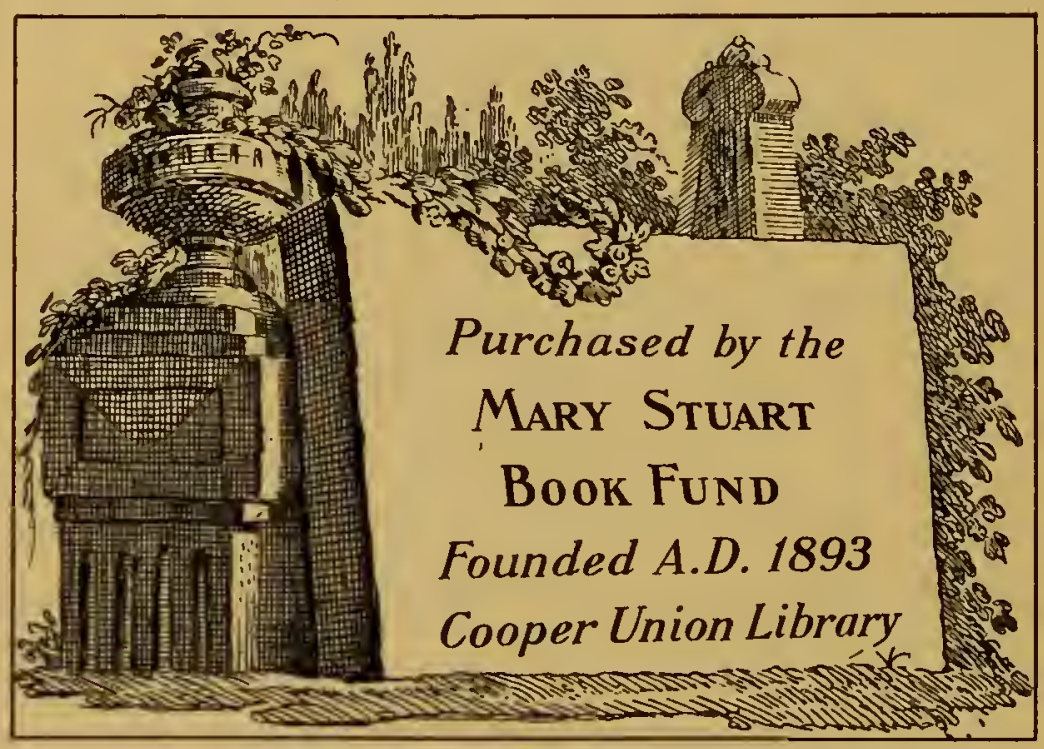





\section{MAKERS OF EARLY AMERICAN SILVER}

ROBERT ENSKO

598 Madison Avenue

NEW YORK CITY 


$$
\begin{aligned}
& \text { Nk } 7112 \\
& 86 x \\
& \text { e+ril }
\end{aligned}
$$

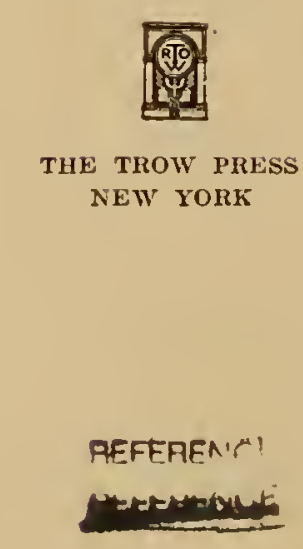




\section{MAKERS OF \\ EARLY AMERICAN SILVER}

S

OME early American pieces of silver are worth more to-day than their weight in gold, yet through the want of knowledge as to their real value and scarcity by some dealers, as well as others, many find their way to the melting pot while the collectors are vainly hunting the cities and towns all over the United States in their efforts to add to their collections. While I obtain numerous pieces from time to time, I can supply but yery little as compared to the demand. I hope this catalogue will help to save some of the old plate. If we had a man like Mr. George Munson Curtis, of the International Silver Company of Meriden, Connecticut, in every state, it would be but a very short time before all that could be known about the makers of silver in the different states would be revealed. He is not only a liberal collector of Connecticut silver but has published a beautifully illustrated book on early silver of Connecticut and a history of its makers, well worth reading. In the exhibitions held recently in the New York and Boston Museums of Art there was a great display of beautiful and artistic workmanship in the early pieces of American silver.

With all the new methods and our machinery we can produce no better silver than the old craftsman who knew every branch of his trade thoroughly. While we find that Massachusetts was the home of the earlier silversmiths, New York, Philadelphia, and Connecticut were also fully supplied with good workmen very shortly after.

On page 27 will be found forty-three pieces of early American plate belonging to Hon. Alphonso T. Clearwater, which he has not been able to fully identify to his own satisfaction. Any information in regard to these marks, such as the names of makers, time and place where they worked, or the owners' names and addresses of pieces bearing the same marks will be gratefully received. I sincerely hope all the marks will soon be fully identified, as it is really too bad to see so many beautiful pieces unidentified.

ROBERT ENSKo

598 Madison Avenue

New York City 



\section{MAKERS OF EARLY AMERICAN SILVER}

IN MANY CASES WHERE THERE IS A RECORD OF BIRTH THE DATE OF WORKING HAS BEEN GIVEN AS TWENTY-ONE YEARS OF AGE

$\begin{array}{ccc} & \text { Connecticut } & \\ \text { Mark } & \text { Ashford } & \\ & \text { Name } & \\ & \text { Russell, Jonathan } & \\ & \text { Berlin } & \\ \text { C. B. } & \text { Beecher, Clement } & \text { Spoons } \\ & \text { Bridgeport } & \end{array}$

Wardin, Daniel

Young, Levi

\section{Brookfield}

Smith, Ebenezer

\section{Brooklyn}

Newbery, Edwin C.

\section{Colchester}

Breed, John

\section{Coventry}

Burnap, Daniel

\section{Danbury}

Blackman, Frederick Starr

Blackman, John Starr

Blackman, John Clark

Clark, Joseph

Mygatt, Comfort Starr

Mygatt, David

Mygatt, Eli

\section{Derby}

Moss, Isaac Nichols

\section{Durham}

\section{Mark \\ Name}

R. Fairchild
Fairchild, Robert

Parmele, James

East Haddam

Foote, William

White, Amos

Enfield

Peabody, John

Terry, Geer

East Hartford

Case, George

Merrow, Nathan.

Pitkin, Henry

Pitkin, John O.

Pitkin, James F.

Pitkin, Walter

\section{Essex}

Pratt, Nathan

Masonic Jewels

Pratt, Nathan, Jr.

Spencer, George

\section{Farmington}

Bull, Martin

Curtis, Lewis

T. N.
Article

Beaker, Tankard (n) 


Connecticut-continued
Guilford
Name

Bushnell, Phineas

S. P.

S. Parmele

Parmele, Samuel

B. W.

Ward, Billious

W. Ward (Seript)

Ward, William

W. W.

W. Ward

\section{Hartford}

Austin, Ebenezer 1764

E. Balch

Baleh, Ebenezer

Spoons

Bartholomew Roswell

Beach \& Sanford

Beach \& Ward

Bedford, J.

Bradley, Richard

Brainard, Charles

Bull, Caleb

Chapin, Aaron

Curch, Joseph

Curch \& Rogers

Dennis, Ebenezcr

Doolittle, Enos

Goodwin \& Dodıl

Goodwin, Allyn

Goodwin, Horace

Goodwin, H. \& A.

Goodwin, Ralph

Greenleaf, David, Jr.

Hilldrup, Thomas

Loud, Asa

Oakes, Frederick

Oakes \& Spencer
Hartford-continued

$\begin{array}{cr}\text { Mark } & \text { Article } \\ \text { Potwine \& Whiting } & \\ \text { Rogers, William } & \\ \text { Sanford, Isaac } & \\ \text { Sargeant, Jacob } & \text { Spoons } \\ \text { Skinner, Elizer } & \\ \text { Spencer, James } & \\ \text { Tiley, James } & \text { Masonic Jewels } \\ \text { Ward, James } & \end{array}$

W. \& B. Ward \& Bartholomew

Bowl Hartford

Ward, Bartholomew \& Brainard

Wells, William

Williams, Drodat

Yeomans, Elijalı

New Hartford

Sadd, Hervey Spoons, Chalice Hebron

Gilbert, Samuel

Welles, Andrew

Young, Ebenezer

Huntington

Lewis, Isaac

Killingworth

Elderkin, Elisha

Ledyard

Gallup, Christopher

\section{Litchfield}

Beach

Beach, Miles

Chalice, Spoons

Merriman, Reuben

Shether \& Thompson

Thomson, Isaac

Ward, IVilliam 


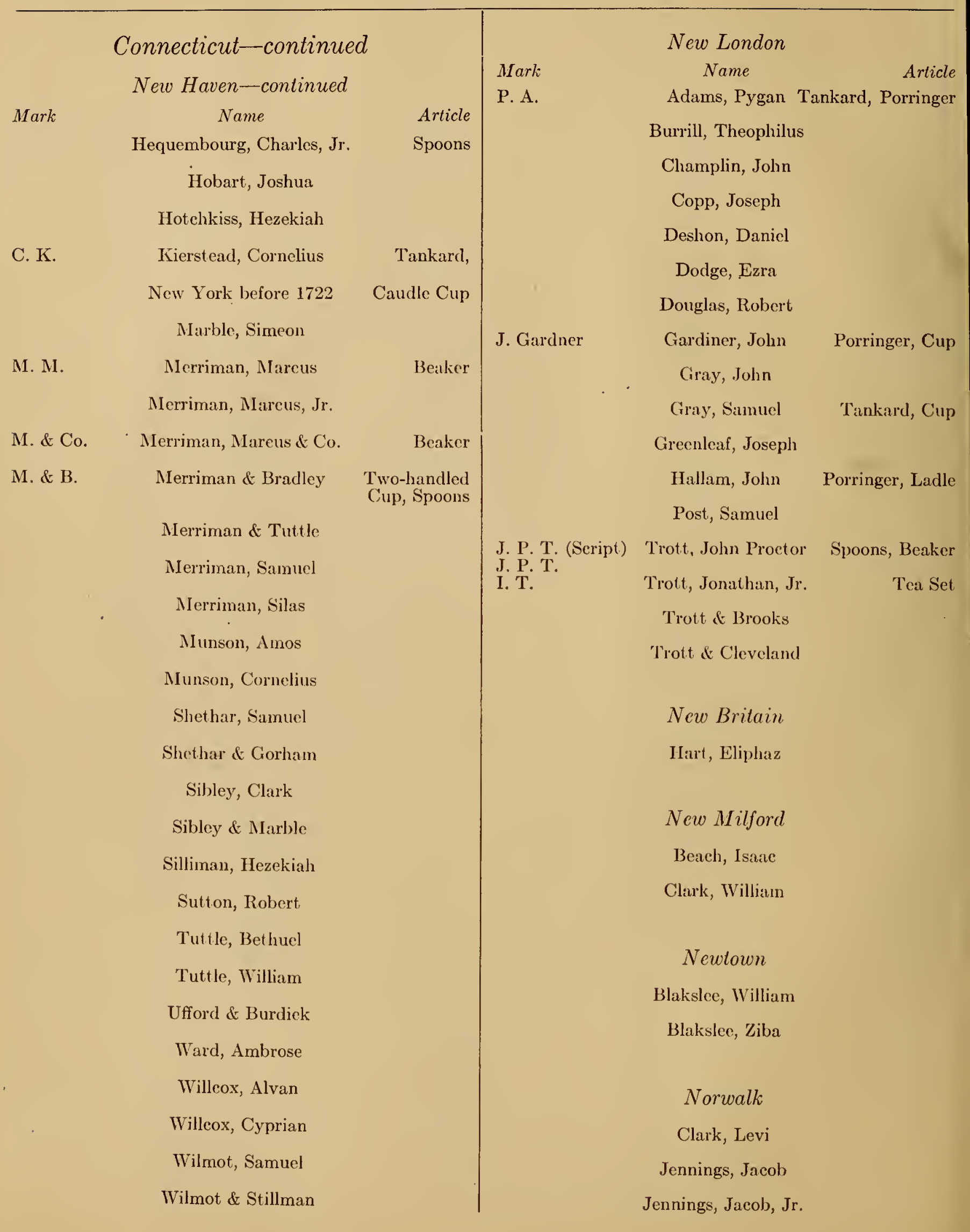




Connecticut-continued
Norwich
Name
Adgate, William
Brewster, Abel
Carpenter, Joseph
Cleveland, William
Coit, Thomas Chester
Coit \& Mansfield
Davison, Barzillai
Dennis, George, Jr.
Greenleaf, David
Grignon, Rene
Gurley, William

Harland, Eagle Harland, Thomas

Harland, Thomas, Jr.

H. \& W., Index Hand Hart \& Wilcox

Spoons

Huntington, Phillip

Huntington, Roswell

Kinney, Thomas

Lathrop, Rufus

Mansfield, Elisha Hyde

Noyes, Samuel

Roath, Roswell Walsten

N. S.

Porringer and

Cover

Shipman, Nathaniel
Mark

.

I. B.

Tracy, Erastus

Tracy, Gordon

Whiting, Charles

\section{Norwalk}

Keeler, Joseph

Rockwell, Thomas

White, Peter

S. H.
Preston

Name

Article

Avery, John

Avery, John, Jr.

Avery, Robert Stanton

Avery, Samuel

Avery, William

Billings, Daniel

\section{Sharon}

Elliott, John Aaron

Stamford

Jarvis, Munson

Reed, Isaac

Stonington

Main, David

Stanton, Daniel

Stanton, Enoch

Stanton, Zcbulon

\section{Stratford}

Benjamin, John

\section{Pepper Castor,} Spoons

\section{Tolland}

Barrows, James Madison

\section{Waterbury}

E. Gunn

Gunn, Enos

Spoons

Holmes, Israel

Hopkins, Jesse

Hopkins, Joseph

Hopkins, Stephen

Tea Spoons

Tompkins, Edmund

\section{Westbrook}

Pratt, Phineas 


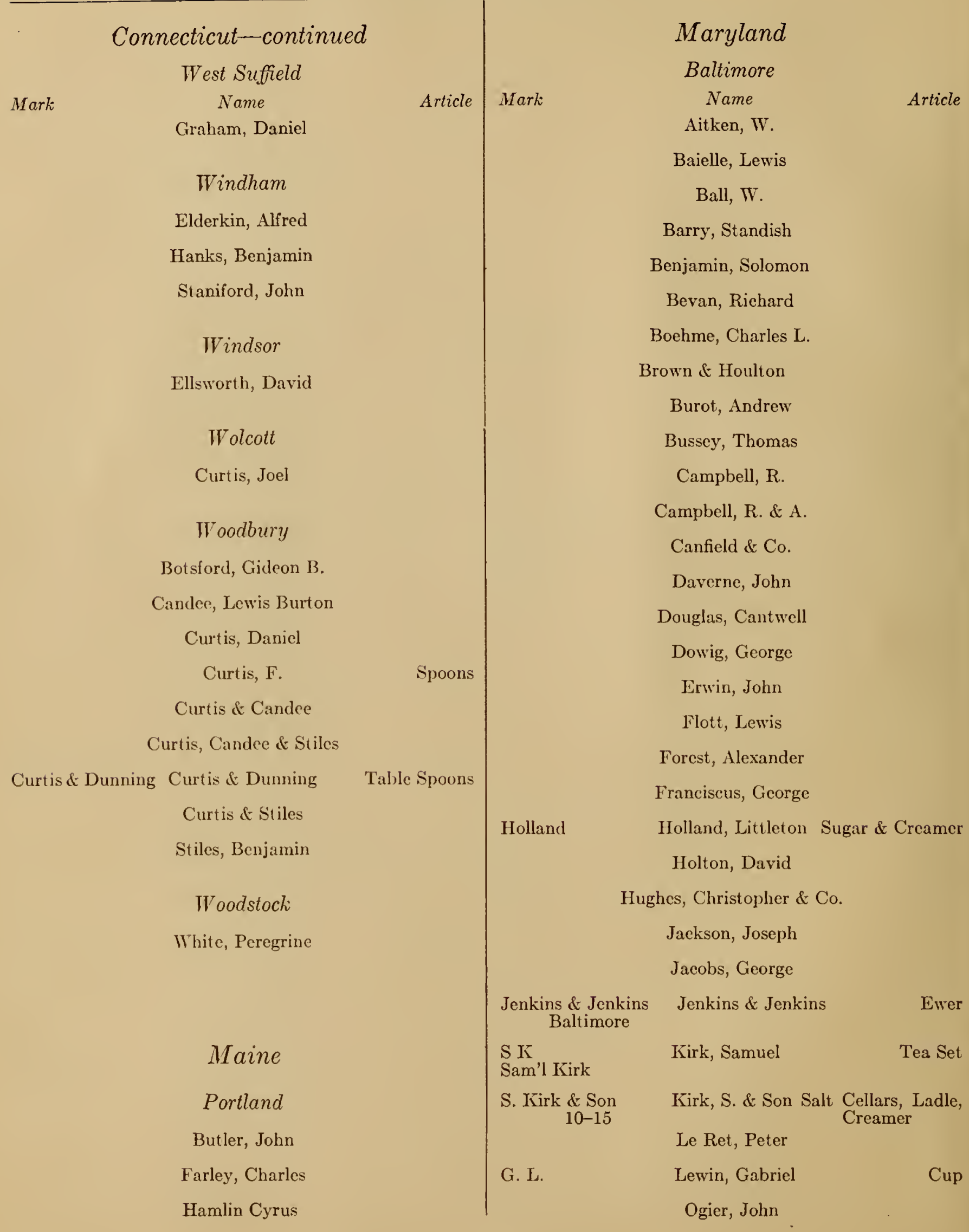




Maryland-continued
Baltimore-continued
Name
Parker, George
Sadtler, Phillip
Sardo, Michael
Smith, John \& Thomas
Stall, Joseph
Stone, Adam
Vincent, Richard

A. E. Warner Warner, Andrew E.

Warner, Andrew E., Jr.

Warner, A. E. \& T. H.

Warner, Thomas $\mathbf{H}$.

Webb, George W.

Webb, James

Wedge, S.

\section{Maryland}

Hamilton, James

Jackson, James

Kenrick, Anwill

Moore, Robert

Wright, Alexander

Massachusetts

Ashby

Edwards, Colvin

\section{Barnstable}

Monroe, John

\section{Boston}

Allen, John

Allen, Thomas

Andrews, $\mathrm{H}$.

N. A.

Beaker
Creamer

Mark

J. Ball

Baldwin \& Jones

S. Baldwin

S. Bartlett

Boyer

I. Bridge
Boston-continued

Name

Ball, John

Arlicle

Mug

Tray

Baldwin, Jabez

Baldwin, $\mathrm{S}$.

Spoons

Barnes, Abraham

Bartlett, S.

Sugar Bowl

Beal, Caleb

Belknap, Samuel

Bentley, Thomas

Bingham, John

Blowers, John

Boyer, Daniel Tea Set, Creamer

Bridge, J.

Flagon

Brinton, Gordon \& Quick

Brigdens, C.

Brown, Ebenezer

Burrill, Joseph

S. Burrill

Burrill, S.

Porringer

Benjamin, Burt. Burt, Benjamin Tea Pot, Porringer

John Burt

Samuel Burt

Burt, John

Brazier

Burt, Samuel

Porringer

Burt, William

Butler, James

L. Cary

Cary, Lewis

Beaker

Churchill, Jesse

Churchill \&

Churchill \& Treadwell

Porringer Treadwell

Clark, Charles

Clark, C. \& G.

Clark, S.

Clark, Metcalf

E. Cobb

Cobb, E.

Tankard

J. Coburn

Coburn, John

Tankard 


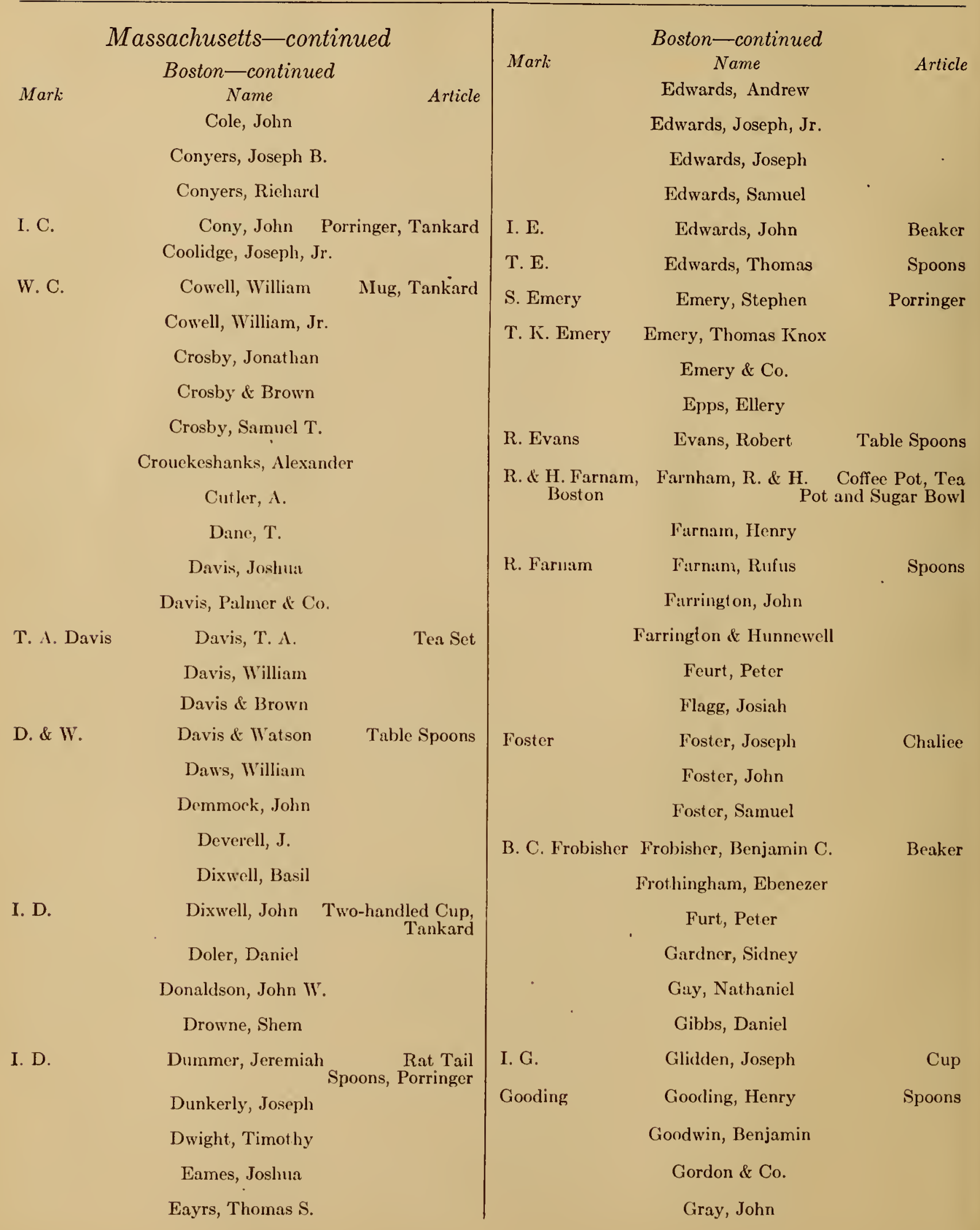




\section{Massachusetts-continued}

Boston-continued

Mark

$$
\text { Name }
$$

Article

Gray, S.

Greene, Benjamin

R. G.

R. Greene

$$
\text { Greene, R. }
$$

Griffith, David

Guille Noah

Haddock, Henry

Haddock \& Andrews

Halstrick, J.

J. Hancock

G. Hanners

Beaker, Mug

Porringer

Hanners, George

Tankard
Grignon, Benjamin

Hanners, George, Jr.

Hansell, Robert,

Harding; Newill

N. Harding \& Co. Harding, N. \& Co.

Harris, Stanwood \& Co.

Haskell, Barnabus

Haugh, Samuel

D. H.

Henchman, Daniel

Hews, Abraham

Hitchborn, Daniel

Hobbs, Nathan

Holyoke, Edward

Homes

Homes, William

Homes, William, Jr.

Horn, E. B.

How, David

Howard, William

Hull, John

Hull \& Sanderson

I. Hurd
Hurd, Jacob
D. I.

Jones, Ball \& Co.

J. B. Jones

Mark

N. Hurd

Jones, Ball \& Poor

\section{Boston-continued}

\section{Name}

Hurd, Nathaniel

Hurst, Henry

Jesse, David

Jones, George B.

Jones, John

Jones, Ball \& Poor

Sugar Tongs, Porringer

Jones \& Ward

I. Kneeland

Salt Spoon

Pure Coin

V.L.\& B.

Kneeland, I. Baptismal Basin,

Keley, Grael

Laforme, F. J.

Laforme, Vincent

Boston

Laforme, Vincent \& Bro.

One-handle

Leach, Charles

Leach, Nathaniel

Legare, Frances
K. Leverett Leverett, Knight

Chalice

Lincoln \& Reed Lincoln \& Reed Boston

Pure Silver Coin

Loring, Henry

I. Loring

Loring, Joseph

Spoons

Low, Francis

Spoons

Low, Ball \& Co.

Low, John J. \& Co.

Luscomb, John G.

J. McF.

MeFarlane, John

Pitcher

Manning, Daniel

Mansfield, John

Mecum, George

Millner, Thomas

Candleabra

Beaker

Tankard
Beaker

Forks

Ewer Tankard

Article

Creamer

Cup 


\begin{tabular}{|c|c|c|c|c|c|}
\hline \multicolumn{3}{|c|}{ Massachusetts-continued } & \multicolumn{3}{|c|}{ Boston-continued } \\
\hline \multirow[b]{2}{*}{ Mark } & Boston-continued & 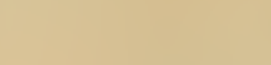 & Mark & Name & Article \\
\hline & Name & Article & P. R. & Revere, Paul & Porringer \\
\hline \multirow[t]{6}{*}{ S. M. } & Minot, Samuel & Creamer & P. R. & Revere, Paul & Tankard \\
\hline & Mitchell, Pheneas & & T. R. & Revere, Thomas & Spoons \\
\hline & Moseley, David & & & Revere \& Son & \\
\hline & Morse, David & & & Rieh, Obadiah & Beaker \\
\hline & Morse, Hazen & & & Ridgeway, James & \\
\hline & Morse, Moses & & & Ridgeway, John & \\
\hline \multirow[t]{3}{*}{ N. M. } & Morse, Nathaniel & Flagon & & Roberts, Frederick & \\
\hline & Morse, Stephen & & & Rogers, A. & \\
\hline & Moses, S. & & & Rogers \& Wendt & \\
\hline \multirow[t]{4}{*}{ Moulton } & Moulton, Ebenezer & $\begin{array}{l}\text { Porringer, } \\
\text { Beaker }\end{array}$ & & Rouse, Michael & \\
\hline & Neyill Richard & & & Rouse, William & \\
\hline & Norcross, Neliemiah & & & Royalston, John & Beaker \\
\hline & Norton, Benjamin & & R.S. & Sinderson, Robert & Bowl \\
\hline \multirow[t]{2}{*}{ I. N. } & Noy's, John & Flagon & & Sargenut, Ensign & \\
\hline & Patddy, Samue] & & & Savage, Thomas & \\
\hline \multirow[t]{8}{*}{ D. P. } & Parker, Daniel & lialle & & Sawin, Silas & \\
\hline & Parkman, J. & & & Shreve, Benjamin & \\
\hline & Paton, A. & & & Simpkins, T. B. & \\
\hline & Pear, Edward & & & Simpkins, Willi:u & \\
\hline & Dopor \& $\mathrm{B}, \mathrm{n}$ & & & Skerry, G. W. & \\
\hline & fetr a Ballin & & 1. Smilh & Stnith, I. & Beaker \\
\hline & Perkins, Houghton & & & & \\
\hline & Pierec, John & & 1. Smith & Smith, I. & Tray \\
\hline \multirow{4}{*}{ B. P. } & Picrpont, Benjamin & Beaker & P. Stacy & Stacy, P. & Flagon \\
\hline & Pollard, William & & & Stanwood, Henry B. & \\
\hline & Pons, Thomas & & & Stanwood, James D. & \\
\hline & Poor, Nathaniel C. & & & Stanwood \& Halstick & \\
\hline \multirow{4}{*}{ I. Potwine } & Potwine, John & Beaker, Tankard & & Stodder \& Frobisher & \\
\hline & Putman \& Low & & & Sutherland, George & \\
\hline & Revere, Edward & & & Swan, Caleb & \\
\hline & Revere, J. II. & & R. Swan & Swan, Robert & Porringer \\
\hline Revere & Revere, Paul & Tea Set & & Symmes, John & \\
\hline
\end{tabular}




\begin{tabular}{|c|c|c|c|c|c|}
\hline Mas & sachusetts-contir & rued & & Hingham & \\
\hline & Boston-continued & & Mark & Name & Article \\
\hline Mark & Name & Article & & Bailey, Loring & \\
\hline & Thomson, Peter & & & Gill, Caleb & \\
\hline J. Trott & Trott, Jonathan & Spoons & & Gill, Leavitt & \\
\hline I. $\mathrm{T}$. & Turner, James & Spoons & & Lincoln, Elijah & \\
\hline A. $\mathrm{T}$. & Tyler, Andrew & Cup & & Norton, Samuel & \\
\hline D. T. & Tyler, David & Nutmeg Grater & & Thaxter, Joseph & \\
\hline & Tyler, George & & & Lowell & \\
\hline & Ward, Richard & & & Sanborn, A. & \\
\hline & Ward, Samuel & & & & \\
\hline & Ward \& Rich & & & Marblehead & \\
\hline & Webb, Barnebus & & & Grant, Thomas & \\
\hline & Wells, George I. & & & Grant, William & \\
\hline A. \& G. Welles & Welles, A. \& G. & Chalice & & & \\
\hline & Wells \& Co. ' & & & Marlboro & \\
\hline B. West & West, B. & Spoons & & Dexter, John & \\
\hline & West Charles & & & & \\
\hline & Whiton, E. & & & Medford & \\
\hline S. Willis & Willis, Stilman & Table Spoons & & Gowen, William & \\
\hline & Willis, T. & & & Milton & \\
\hline E. W. & Winslow, Edward & Tankard & & Davenport, Samuel & \\
\hline & Woodward, Eli & & & & \\
\hline & Woodward \& Grosjean & & & Nantucket & \\
\hline & Charlestown & & & Barrett, S. & \\
\hline I. A. & Austin, Josiah & Tray & $\begin{array}{l}\text { J. Easton, } \\
\text { Nantucket Pure Coi }\end{array}$ & Easton, James & Spoons \\
\hline $\begin{array}{l}\text { Z. Brigden } \\
\text { Z. B. }\end{array}$ & Brigden, $\mathrm{Z}$. & $\begin{array}{r}\text { Teapot, Pepper } \\
\text { Castor }\end{array}$ & & Easton, Nathaniel & \\
\hline Chas. Faris & Faris, Charles & Tea Set & $\begin{array}{l}\text { Easton \& Sanford, I } \\
\text { Nantucket }\end{array}$ & Easton \& Sanford & Spoons \\
\hline & Deerfield & & & Sanford, F. S. & \\
\hline & Parker, Isaac & & & New Bedford & \\
\hline & Harwich & & & Delano, Jobez & \\
\hline & Nickerson, Baty & & & Pitman, William R. & \\
\hline
\end{tabular}




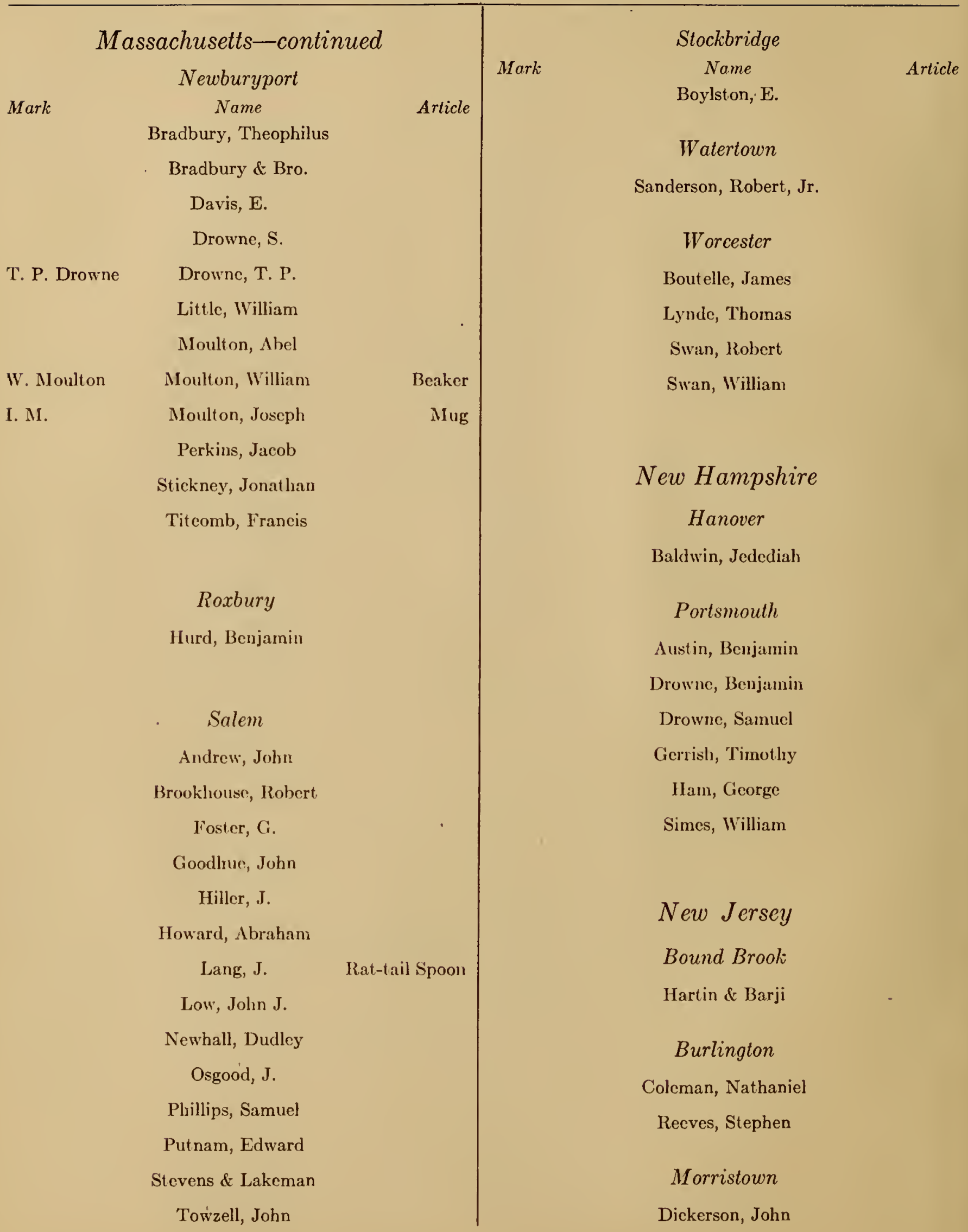




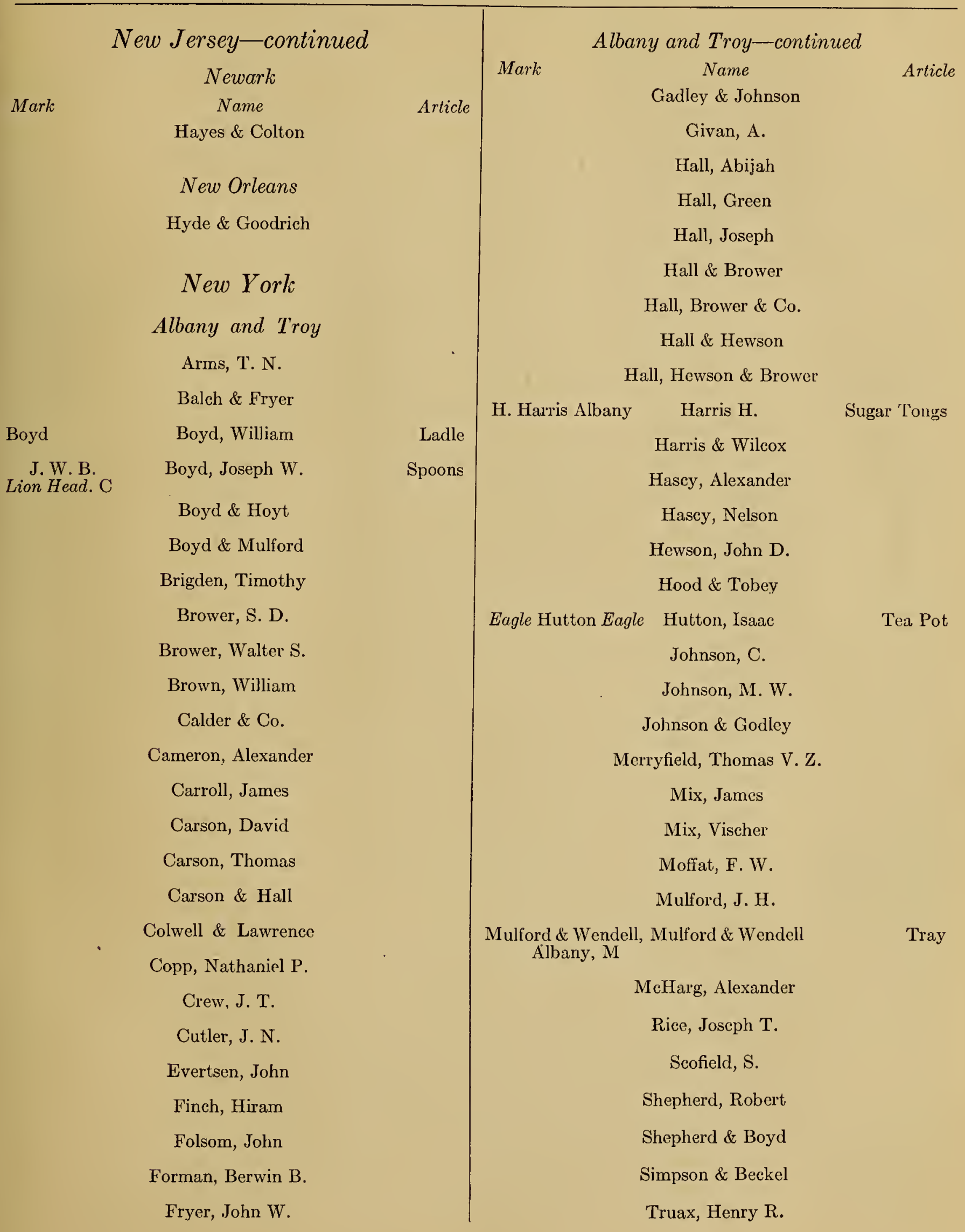




\begin{tabular}{|c|c|c|}
\hline \multirow{2}{*}{\multicolumn{3}{|c|}{$\begin{array}{c}\text { New York-continued } \\
\text { Albany and Troy-continued }\end{array}$}} \\
\hline & & \\
\hline \multirow[t]{3}{*}{ Mark } & Name & Article \\
\hline & Van Bergen, John & \\
\hline & Waterman, George & \\
\hline \multirow{6}{*}{$\begin{array}{l}\text { Wendell \& } \\
\text { Roberts }\end{array}$} & Wendell \& Roberts & Vases, Spoons \\
\hline & Wilson, Albert & \\
\hline & Ithaca & \\
\hline & De Remicr \& Mead & \\
\hline & Mead, Adriance \& Co. & $y$ \\
\hline & New York City & \\
\hline \multirow[t]{11}{*}{ W. Adams } & Adams, William & Bcaker \\
\hline & Acklerly, F. M. & \\
\hline & Allison, Peter & \\
\hline & Alstyne, Jeronemus & \\
\hline & Anderson, William & \\
\hline & Andrews, J. & \\
\hline & Anthony, $W$. & \\
\hline & Archie, John & \\
\hline & Backus, D. & \\
\hline & Bailey, Simcon A. & \\
\hline & Baker, Anson & \\
\hline $\begin{array}{l}\text { Ball, Tompkins } \\
\text { \& Black } \\
\text { Patent, } 1846\end{array}$ & $\begin{array}{c}\text { Ball, Tompkins \& Black } \\
\text { (Successors to } \\
\text { Marquand \& Co.) }\end{array}$ & $\begin{array}{r}\text { Cheese Scoop, } \\
\text { Tea Set }\end{array}$ \\
\hline \multirow[t]{4}{*}{ A. B. } & Bancker, Adrian & Spoons \\
\hline & Barret, James & \\
\hline & Batchellor, N. & \\
\hline & Bay, A. S. & \\
\hline \multirow[t]{2}{*}{ Bayley } & Bayley, S. H. & Sugar Bowl \\
\hline & Bayley \& Douglas & Sugar Bowl \\
\hline I. Benedict & Benedict, J. & Tablespoon \\
\hline $\begin{array}{l}\text { A. C. Benedict, } \\
28 \text { Bowery }\end{array}$ & Benedict, A. C. & Sugar Tongs \\
\hline Benjamin & Benjamin, B. & Sugar Tongs \\
\hline
\end{tabular}

\section{New York City-continued}

Mark Name Arlicle Bennet, James

Berkenbush, C. H.

Besley, Thouvet

Bigotat, S.

Boelen, Jacob

Bogardus, Everdus

N. Bogert

Bogert, N.

Shcaf of Wheat

Tablespoons

Bogert, Albert

Bolton, James

Bourdet, Stephen

Bowder, I.

Bowne, Samuel

G. B.

G. Boyce

Boyce, G. Tody Spoon, Ladle, 2 Mugs, Bowl

Boyce, J.

Brady, William V.

Brock, John

E. B.

Braslıer, Ephraim Teaspoons, Sugar Tolugs

\section{Brevoort, John}

Brinkley, William

Broadhurst, Samuel

Brower \& Rusher

Brown, Samuel C.

Bruff, C. O.

Bryne, John

Bryne, James

Burger, N. York Burger, John Tablespoons, Sugar D. I. Burger, U. Burger, D. I. Bowl

Cady, Samuel

Cady \& Bachus

Caldwell, E.

Canavillo, S. 


\section{New York-continued}

\section{New York City-continued}

Mark Name

Article

Cann, John

Cant, Goedfrey

Caralin, Pierce

Carman, John

Carroll, John

Caston, Francoise

Chandless, William

Charters, James

Charters, Cann \& Dunn

Chalice

Chase, J. D.

Chene, Daniel

Chitry, P.

Clapp \& Riker

J. H. Clark

Clark, J. H.

- Mustard Spoon

Clark \& Anthony, Clark \& Anthony Spoons, Ladle Lion Head

Coen, Daniel

Cole, Albert

Coley, W.

Coley, Simeon

Colton, Levi

Cook, I.

B. \& J. Cooper

Cooper, B. \& J.

Sugar Bowl

Cooper, B.

Cooper, John

F. W. Cooper $\frac{900}{1000}$ Cooper, F. W.

Cornelison, C.

Crawford, John

Dalley \& Halsey

Dawson, John

Denise, J. \& T.

De Peyster, William

Mug
New York City-continued

Mark

Name

Article.

De Perrigang, Otto

De Remier, Peter

Dobbs, Adam

Dodge, E.

Douglas, J.

Douglas, Alexander

Dubois, J.

Duche, B. R.

C. Dunn

Dunn, Cary Tankard, Creamer

C. D. 13

Edmechat, C.

T. Edwards

Edwards, Thomas

Tray

Ellison, Peter

Eoff, G.

Spoons

G. Eoff

Eoff \& Connor

Eoff \& Phyfe

Etting, Benjamin

Evans, John

Fielding, George

Forbes, A. G.

Forbes, B. G.

Forbes, C. V. G. Soup Ladle

G. Forbes

Forbes, Garrit

Tea Set, Cream Jug, Sugar Tongs

I. W. F., I. W. Forbes, Forbes, I. W. Butter Knives, New York, Anchor, Head, and Star

W. G. Forbes

Forbes, W. G.

Salt Spoons

W. Forbes

Forbes, William Tablespoons,
Tea Set

Fourniquet

Fourniquet, Lewis

Sugar Bowl

Fowler, G.

N. Francis, Eagle Francis, N. Spoons, Sugar Tongs, Fueter, Daniel

Fueter, Daniel C. 
New York-continued

New York City-continued

Mark Name

Article

Fueter, Lewis

J. L. Gale

Gale, John L.

Sugar Bowl

Gale, William

Wm. Gale, Jr. Gale, William, Jr. Pair Candlesticks Gale, IV. \& Son

G. W. \& H. Gale, Wood \& Hughes

Tea Set

G. H. ${ }^{1}{ }^{7}$

Gale \& Hayden Mug, Cream Piteher

Gale \& Stickler

Gale \& Willis

Gale of Willis

Punch Ladle, Comport

B. Ciardner, Now York

Gaulner, B. Tea Set, Spoons

B. C., Lion IIead, C.

Giarrisson, J.

Ciclston, G. S.

Gelston \& Treadwell

Gelst on \& Co., New York

Gelston \& Co.

Gilbert, William

Gilbert \& Cunningham

Goelet, Phillip

Gorion, A. \& J.

Gordon, Andrew

Gordon, J.

Gough, James

Griffing, C.

Grigg, W.

Gmnee, B. \& S.

Hall, Drew

1. Halsey

Halsey, Jabez

Tea Pot

Halstead, Benjamin

Hamersley, Thomas

Harper, T. W.
New York City-continued

Mark

Name

Hart, J.

Hastier, John

Hastier, Marquette

Hayden \& Gregg Hayden \& Gregg

Tray

Hays, Andrew

Heath, Jolin

Hebbard, H.

Hendrickse, A.

Heron, Isaac

Herbert, T. B.

Huertin, William

IT. B. H.

Head, Lion, G.

Heyer, W. B. Basket of Fruit, Sugar Tongs, Spoons

Hinsdale, E.

Holmes, J.

Hutton, Jolnn

Jackson, John

Jackson, Daniel

Johnson, Samuel

Keith, $\mathrm{T}$.

Sugar Tongs

Kendall, C.

Ǩidney, Cann \& Johnson

C. K

Kierstede, Cornelius

Candle Cup, Baptismal Basin

Kingston, John

Kimberly, W.

Kip, Benjamin

Ledell, Joseph

Lent, John

Le Roux, Bartholomew

Le Roux, Charles

Le Roux, John

Lockwood, F. 
New York-continued

Mark

\section{New York City-continued} Name

Article

Luzerder, Benjamin

Lyell, David

Lyng, John B.

Maldrid \& Co.

Marquand, Frederick

Marquand

Marquand \& Co.

Marquand \& Co. Creamer, Ewer Martin, $\mathbf{P}$.

Maverick, Peter R.

Maverick, Peter

McClymon, J. C

Mecum, John

Merkler, John H.

Merriman, C.

Milne, F.

J. C. M.

Star, Anchor, M.

Moore, J. C.

Moore \& Brown

Moore, E. C.

Morris, John

Morris, Sylvester

Mott, W. \& J.

I. M.

Moulinar, John

Meyers, Meyer

Mudge \& Co.

Newkirke, J.

Onclebag, Gerret

Overin, Richard

Parisien, Otto

Parisien \& Son

Pattit, Thomas

Pearce, Samuel

Pearson, John

Tea Pot

Tea Set
New York City-continued

Mark

Name

Article

Pelletrau, E.

Pinto, Joseph

Platt, George W.

Platt \& Brother

Tea Set

Polhamus, J.

Portram, Abraham

Quintard, Peter Beaker, Tankard

S. Richard,

New York

Richard, S.

Mug

Richardson, Thomas

G. R.

Ridout, George

P. Riker, New York Riker, P.

Candlesticks

Riker \& Alexander

Ritter, Michael

Robert, Christopher

Roberts, Michael

Rockwell, E.

Roe, W.

Rominie, John

Roosevelt, N.

Roshore, John

Roshore \& Prime

Russell, John H.

Sanderson, W.

I. Sayre, New York Sayre, Jocl

Schaats, Bartholomew

Schenck, Garad

Sell, J.

Sexnine, Simon

Schenck, John

Singleton \& Young

Skinner, Abraham

T. S.

Skinner, Thomas

Mug 
New York-continued

Mark

New York City-continued

Slydell, Joshua

Smith, James

Smith, William

Sougue, M.

Soumine, Simeon

Squire \& Bros.

Staats, Bartholomew

Staples, John J., Jr.

Stebbins \& Co. Stebbins \& Co.

Stebhins \& Howe

Stephens, George

Steven, George

Stewart, C.

Stollenwerek \& Co.

Stone \& Osborn

Stout enburgh, T.

Stuart, H.

T. \& P. T.

Article

Mark
D. V. V.

New York City-continued
Van Voorhis, Daniel
Sugar Bowl
Van Voorhis \& Cooley
Van Voorhis \& Son
Vergereau, $P$.
Vernon, J. \& Co.
Vernon, John
Wenman, B.
Whitlock, Thomas
Wilson, $\mathrm{R}$.
Windover, J.

H. Wishart,

New York

Wishart, $\mathrm{H}$.

Coffee Pot Eagle

Woods

IV. \& Hughes

Woods, Freeman

Tea Pot

Wood, J. E.

Woor \& Hughes Salt Cellars and Spoons, Ladle and Tongs, Forks and Spoons, Cups and Mugs

IVool, J. IV.

Wynkoop, C.

Ten Eyck, C.

Thomas, W.

Thomson, James

Thomson, W.

Tingley, Samuel

Underhill, A.

Underlill, Thomas

Underhill \& Vernam

Van Buren, P.

Van Buren, William

Vanderspirgel, J.

Van Dyke, Peter

Van Dyke Richard

Wyneoope, B.

Tankard

\section{Pennsylvania}

\section{Lancaster}

Becker, Phillip

Hall, Charles

\section{Sugar Bowl}

Battels, A. T.

Butler, N.

Leach \& Bradley

Storrs \& Cooley

Price, John 
Mark

J. Aitken

\section{Pennsylvania-continued}

Philadelphia

$$
\text { Name }
$$

Aitken, John

Article

Alexander, Samuel

Alford, Samuel

Alford, Thomas

Allen, James

Allen, Robert

Andrews, J.

Andrews, Henry

Anthony, J.

Anthony, Joseph \& Son

Anthony, J.

Armstrong, John

Ashmead, William

Atherton, Nathan, Jr.

Baily, John

Bailey, John

Bailey \& Kitchen

W. Ball

Ball, William

Bard, J.

Creamer

J. Bard

Bard \& Hoffman

Bard \& Lamont

Bard, C. \& Son

Bartram, William

Berard, Andrew

Best, Josiah

Black, Jolin

Blondel, Anthony

Boudinot, Elias

Bright, Anthony

Brown, John

Bruff, I.

Philadelphia-continued

Mark Name Article

Bumm \& Shepper

Burdock, Nicholas

Butler \& McCarty

Campwell, W.

Cario, Michael

Carmon, John

Childs, George K.

Cooke, Joseph

Cumming, David B.

J. Curry, Phia

Curry, John

Beaker

Curry \& Preston

I. D., Philadelphia David, John Spoons, Ladle

David, Peter

David, Lewis A.

Davy, Adam

Dickerson, John

Dorsey, Joshua

Dowig, George

Drewrey, G.

Dubois, A.

Dumontet, S. B.

Dunlevey, Robert

Dumorte, John

Dupuy, Daniel, Jr.

D.D D.D

Dupuy, Daniel

Sugar Bowl, Skewer, Table Spoons

England, William

Faber, William

Faber \& Hoover

T. Fletcher, Philad. Fletcher, T

Tea Set

Fletcher \& Gardner

Fling, George 


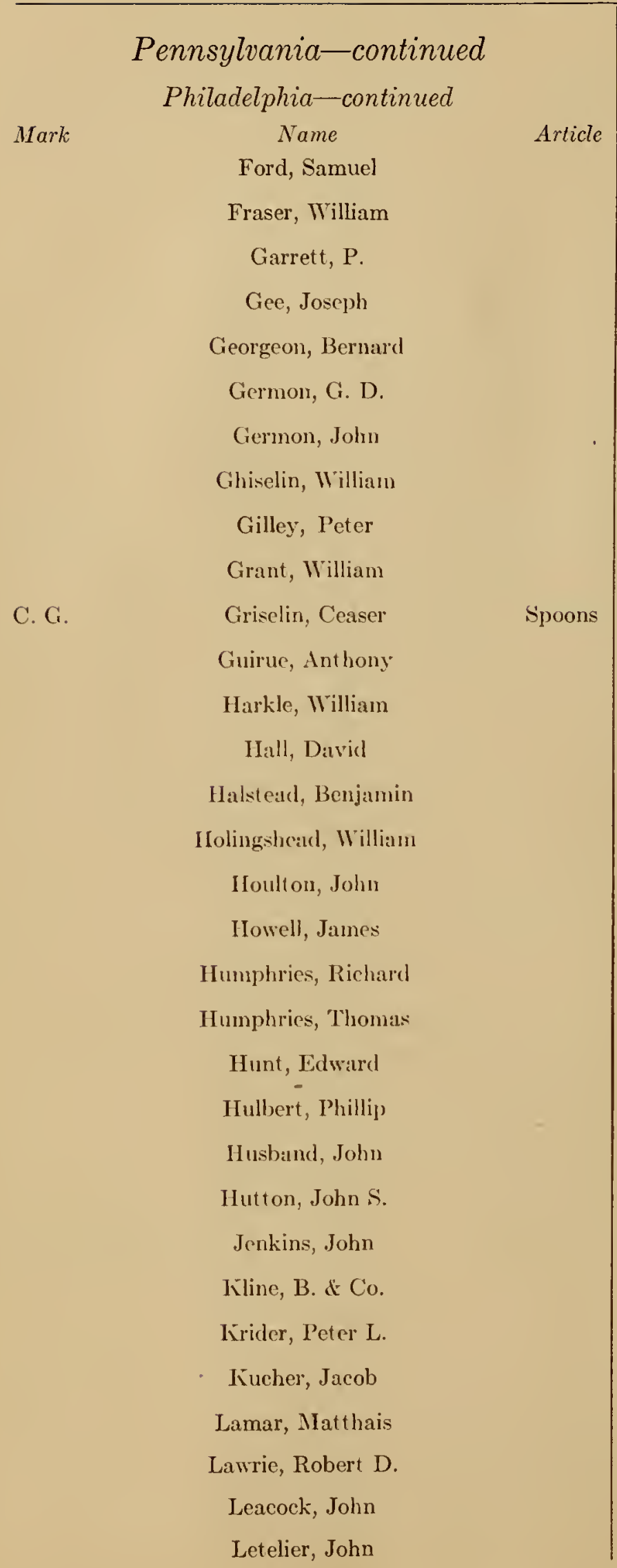

Mark

H. Lewis

J. Lownes

I. Myers

Anty. Rasch

S. Richards
Philadelphia-continued

\section{Name}

Article

Lewis, Harvey

Tea Set

Lewis \& Smith

Lownes, Edward

Lownes, Joseph

Tea Pot

Lownes, J. \& J. H.

Lyng, John

Marshall, Joseph

McFee, John

McMullen, John

MrcMullin \& Black

Miles, John

Milne, Edward

Musgrave, James

My"ers, John

Spoons

Olivier, Peter

Perraux, Petel

Pinchin, William

Pitt, Richard

Poinergnon, Francis

Poissonier, F.

Polgrain, Quom

Poupard, James

Price, Benjamin

Rasch, Anthony

Tea Set

Rasch \& Willig

Reed, Osman

Reeder, Abner

Rich, Joseph

Richards, Samuel

Creamer

Richards \& Williamson

Richardson, Francis

Richardson, Joseph

Richardson, J. 


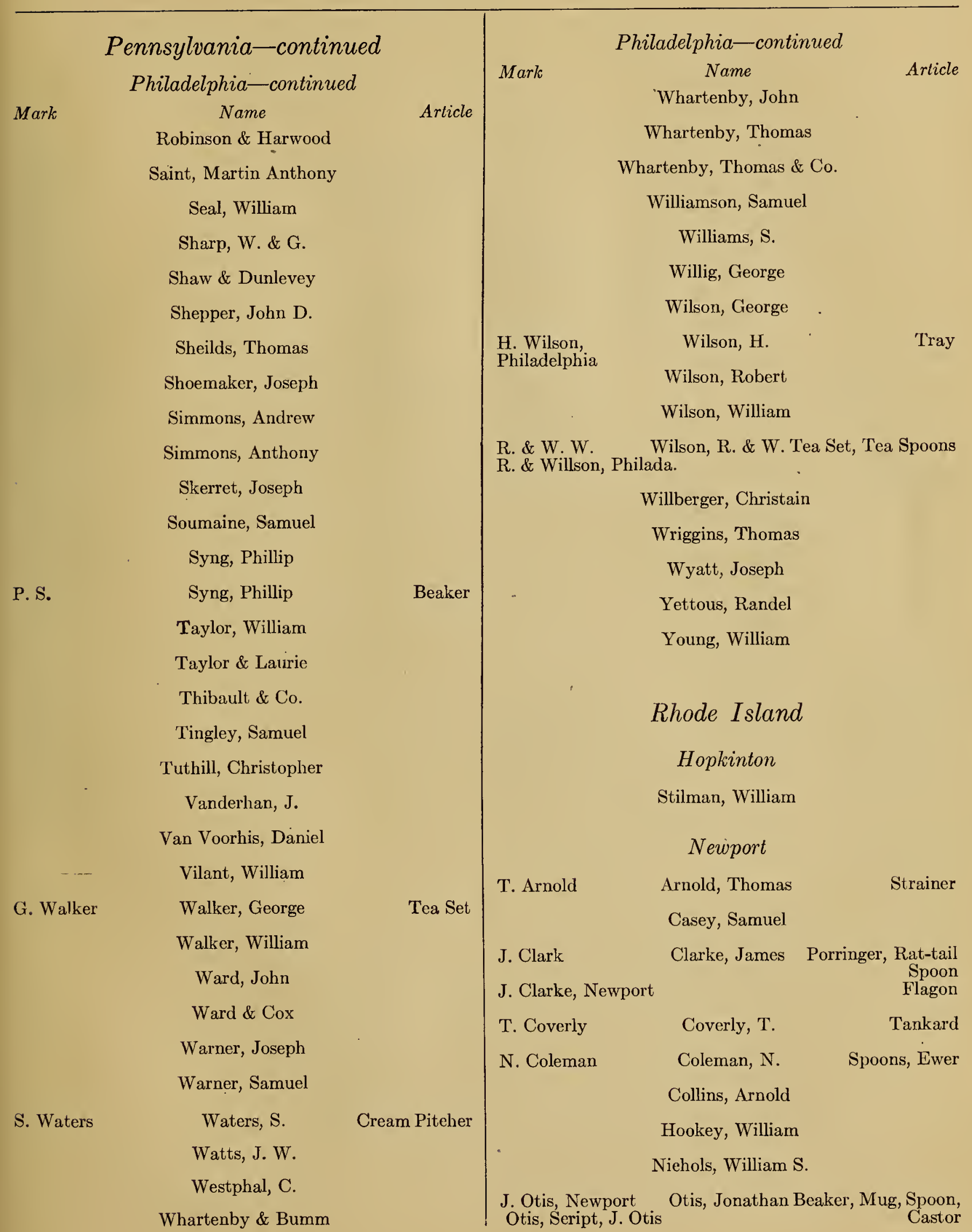




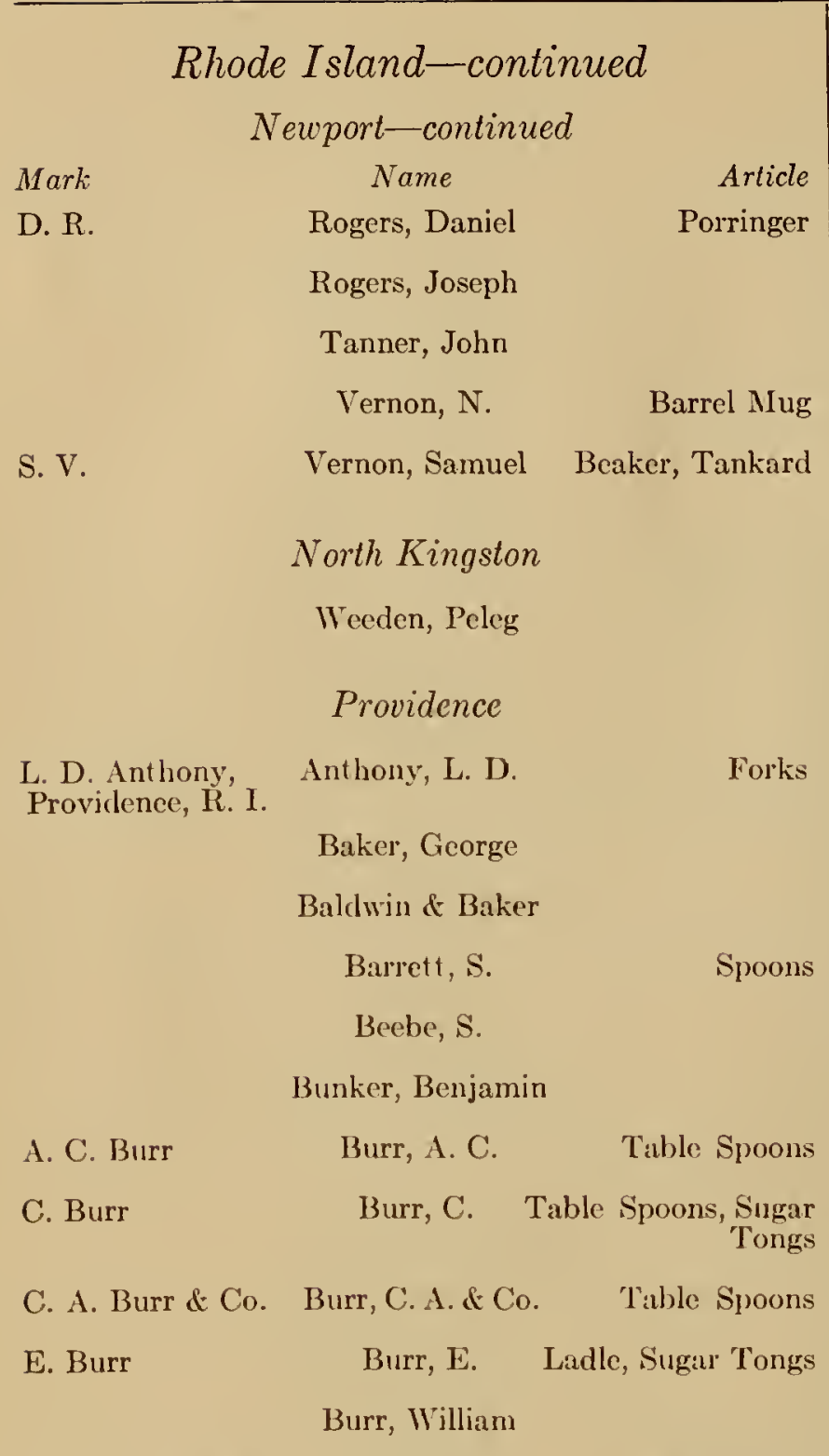

A. C. Burr S. W. Lee Burr \& Lec

Table Spoons

Clark, Gcorge D.

Crandell, Benjamin

Davis \& Babbitt

Davis, Samucl

Dodge, Cyril

Dodge, Nehemiah

Frost \& Mumford

Gafkins, J. Spoons and Ladle.

Gibbs, John

Gorham, Jabez

\section{Providence-continued}

Mark

Name

Article

Gorham \& Webster

Gorham \& Son

Gorlham \& Thurber Gorham \& Thurber Gorham \& Thurber, G.,

Providence, R. I.

Greene, William \& Co.

Hadwen William

Kielley, Allen

Iielley, E. G. \& J. H.

S. W. Lec

Lee, S. IV.

Table Spoons

Jenckes, John C.

Mumford, H. G.

Nichols, Bassett

Pitman \& Dorrance

Pitman, Saunders

Pitman \& Dodge

Pitman, John K.

Richmond, G. \& A.

Sackett \& Willard

Veazie, Joseph

Vinton, David

Webster, H. L.

Whitaker \& Green

IVilliams, Stephen

South Kingston

Cascy, Gideon

Westerly

Bradford, Charles H.

Soup Ladle, Spoons 
Unidentified makers and marks of American silver in Hon. A. T. Clearwater's Collection. Any information in regard to same will be gratefully received.

Robert Ensko.

\section{A.T.C.Collection Marks}

$\begin{array}{ll}\text { Tankard } & \text { G. F. in Oval twice } \\ \text { Another } & \text { I. G. L. in half oval, four times } \\ \text { Mug } & \text { L. A. in Cartouche } \\ \text { Another } & \text { Phillip Alexander, large block } \\ \text { Beaker } & \text { L. A. in rectangle, } 3 \text { times } \\ \text { Another } & \text { S. D. in shaped shield, twice } \\ \text { Bowl } & \text { B. E. in rectangle } \\ \text { Another } & \text { R. E. in rectangle } \\ \text { Sugar Bowl } & \text { T. T. with Crown above, twice } \\ \text { Can with Cover } & \text { R. G. in oval } \\ \text { Saucepan } & \text { St. Lars in shaped rectangle } \\ \text { Another with Lid } & \text { H. P. Head + all in squares } \\ \text { Coffee Pot } & \text { J. A. in lobed shield } \\ \text { Tea Pot } & \text { I. F. S. in rectangle } \\ \text { Another } & \text { C. \& N. } \\ \text { Another } & \text { I. A. in shaped shield } \\ \text { Porringer } & \text { A. W. L. three times } \\ \text { Tray Candlesticks } & \text { B. M. in square } \\ \text { Jitcher } & \text { I. A. in oval } \\ \text { A. in script in oval, twice }\end{array}$

Another J. A. in shaped shield twice

Another

W. L. in shaped rectangle

Another

P. Prie in shaped shield

Creamer

E. D. lion, rampant, twice

Salt Cellar

C. C. K. in rectangle, F. in oval

Another

C. A. in small rectangle

Trencher Salt

J. W. in oval

Two more
I. + P.
A.T.C.Collection Marks

Ladle

I. N. twice

Another

Cup

W. H. in linked letters, small rectangle

P. J, in heart-shaped shield

Taper Box D. in script in circle

Box

A. L. in Cartouche

Patch Box

I. W. in oval

Two Knee Buckles

F. B. in shaped shield

Two Shoe Buckles T. A.

Two more

S. A. in oval

Pepper Caster

$J$. B. in monogram script in circle

Nutmeg Grater

I. K.

Butter Tester

T. K. twice

Another

T. H.

Wine Funnel Rest R. K. in square, three times

L. S. in squares

Brazier

C. lion rampant, in rectangle

Information in regard to the following names will be appreciated by several collectors:

Clarici, J,

Crittenden, N. E., about 1790

Gaither, J.

Gaskins

Ginochio, J. B.

Gouiran, Mel

Greenwood, C. F.

Lynch, J.

Moses, O. H.

I. NR., Phia., 1776

Rae, W. J.

Tenny, 251 Broadway, New York

Tucker, J. W., Pearl St., New York 


\begin{tabular}{|c|c|c|c|c|c|c|c|c|c|}
\hline Name & Born & Working & Died $P$ & Page & Name & Born & Working & Died & Page \\
\hline Aekerly, F. M. & & 1800 & & 18 & Arnold, Thomas & & 1780 & & 25 \\
\hline Adams, Pygan & 1712 & 1735 & 1776 & 8 & Ashmead, William & & 1797 & & 23 \\
\hline Adams, William & & 1833 & & 18 & Atherton, Nathan, Jr. & & 1824 & & 23 \\
\hline Addison, George M. & & 1804 & & 23 & Atterbury, J. & & 1799 & & 7 \\
\hline Adgate, William & 1744 & 1765 & 1779 & 9 & Austin, Benjamin & & 1775 & & 16 \\
\hline Aitkin, John & & 1797 & & 23 & Austin, Ebenezer & 1733 & 1754 & & 6 \\
\hline Aitkins, W. & & 1802 & & 10 & Austin, Josiah & 1719 & 1748 & 1780 & 15 \\
\hline Alexander, Samuel & & 1797 & & 23 & Austin, Nathanjel & & 1789 & & 11 \\
\hline $\begin{array}{l}\text { Alexander, S. \& A. Sim- } \\
\text { mons }\end{array}$ & & 1800 & & 23 & Avery, John & & 1762 & 1794 & 9 \\
\hline Alford, Samuel & & 1759 & & 23 & Avery, John, Jr. & 1755 & 1776 & 1815 & \\
\hline Alford, Thomas & & 1762 & & 23 & $\begin{array}{l}\text { Avery, Robert Stanton } \\
\text { Avery Samuel }\end{array}$ & $\begin{array}{l}1771 \\
1760\end{array}$ & 1794 & 1846 & 9 \\
\hline Atlen, John & & 1699 & & 11 & $\begin{array}{l}\text { Avery, samuel } \\
\text { Avery, William }\end{array}$ & 1765 & $\begin{array}{l}1781 \\
1786\end{array}$ & $\begin{array}{l}1836 \\
1798\end{array}$ & 9 \\
\hline Allen, Jancs & & 1720 & & 23 & Babcock, Samuel & $17 S S$ & $1 S 12$ & 1857 & 7 \\
\hline Allen, Robert & 1755 & 1787 & 1825 & 23 & $\begin{array}{l}\text { Backus, D. } \\
\text { Baielle, Lewis }\end{array}$ & & 1792 & & 19 \\
\hline Allen, Thomas & & 1758 & & 11 & $\begin{array}{l}\text { Batene, Lewis } \\
\text { Bailey, Henry }\end{array}$ & & 1789 & & 11 \\
\hline Allison, Peter & & 1792 & & 18 & Bailey, John & & 1762 & & 23 \\
\hline Alst yne, Jeronimus & & 1787 & & 18 & Bailey, John & & 1783 & & 23 \\
\hline Andrew, John & 1747 & 1769 & 1791 & 16 & Bailey \& Kitehen & & 1850 & & 23 \\
\hline $\begin{array}{l}\text { Andrews, } \mathrm{H} \text {. } \\
\text { Andrews, Henry }\end{array}$ & & 1796 & & 23 & Bailey, L. & & 1780 & & 15 \\
\hline Andrews, J. & & 1810 & & 23 & Baily, Benjamin & & & & 11 \\
\hline Andrews, J. & & 1790 & & 18 & Baily, Dimeon A. & & & & 18 \\
\hline Anderson, Williarn & & 1746 & & 18 & Baker, Anson & & 1825 & & 18 \\
\hline Anthony, Joseph \& Son & & 1811 & & 23 & Balch \& Fryer & & 1784 & & 17 \\
\hline Anthony, J. & & 1810 & & 23 & Balch, Ebenezer & 1723 & 1784 & 1808 & 6 \\
\hline Anthony, J. & & 1770 & & 23 & Balclwin, Jabez & & 1813 & & 11 \\
\hline Anthony, L. D. & & 1780 & & 26 & Baldwin \& Jones & & 1813 & & 11 \\
\hline Anthony, W. & & 1800 & & 18 & Baldwin, Jedediah & & 1790 & & 16 \\
\hline Archie, John & & 1759 & & 18 & Baldwin, S. & . & 1810 & & 11 \\
\hline Armstrong, John & & 1811 & & 23 & Baldwin \& Baker & & 1817 & & 26 \\
\hline Arms, T. N. & & 1849 & & 17 & Ball, John & & 1765 & & 11 \\
\hline
\end{tabular}




\begin{tabular}{|c|c|c|c|c|c|c|c|c|c|}
\hline Name & Born & Working & Died & Page & Name & Born & Working & Died I & Page \\
\hline Ball, William & & 1752 & & 23 & Benedict, A. C. & & 1840 & & 18 \\
\hline Ball, Tompkins \& Black & & 1846 & & 18 & Benedict, J. & & 1830 & & 18 \\
\hline Ball, W. & & 1802 & & 10 & Benjamin, B. & & 1825 & & 18 \\
\hline Baker, George & & 1825 & & 26 & $\begin{array}{l}\text { Benjamin, Barzellai } \\
\text { Benjamin, Everard }\end{array}$ & $\begin{array}{l}1774 \\
1807\end{array}$ & $\begin{array}{l}1815 \\
1829\end{array}$ & $\begin{array}{l}1844 \\
1874\end{array}$ & 7 \\
\hline Bancker, Adrian & & 1792 & & 18 & Benjamin, John & 1730 & 1750 & 1796 & 9 \\
\hline Bard, C. \& Son & & 1850 & & 23 & Benjamin, Samuel C. & 1801 & 1819 & 1831 & 7 \\
\hline Bard \& Hoffman & & 1837 & & 23 & Benjamin, Solomon & & 1817 & & 10 \\
\hline Bard, J. & & 1800 & & 23 & Bennet, James & & 1769 & & 18 \\
\hline Bard \& Lamont & & 1841 & & 23 & Bentley, Thomas & & 1796 & & 11 \\
\hline Barnes, Abraham & & 1716 & & 11 & Berard, A. & & 1797 & & 23 \\
\hline Barrett, James & & 1805 & & 18 & Berkenbush, C. H. & & 1825 & & 18 \\
\hline $\begin{array}{l}\text { Barrows, James Madi- } \\
\text { son }\end{array}$ & & 1828 & & 9 & $\begin{array}{l}\text { Besley, Thauvet } \\
\text { Best, Josiah }\end{array}$ & & $\begin{array}{l}1727 \\
1723\end{array}$ & & $\begin{array}{l}18 \\
23\end{array}$ \\
\hline Barry, Standish & & 1790 & & 10 & Bevan, Richard & & 1804 & & 10 \\
\hline Barrett, S. & & 1800 & & 15 & Bigotut, S. & & 1800 & & 18 \\
\hline $\begin{array}{l}\text { Bartlett, S. } \\
\text { Bartram, William }\end{array}$ & & 1769 & & 23 & Billings, Daniel & & 1795 & & 8 \\
\hline Bartholomew, Roswell & 1781 & 1804 & 1830 & 6 & Black, John & & 1819 & & 23 \\
\hline $\begin{array}{l}\text { Batchellor, N. } \\
\text { Battels, A. T. }\end{array}$ & & 1847 & & $\begin{array}{l}18 \\
22\end{array}$ & $\begin{array}{c}\text { Blackman, Frederick } \\
\text { Starr }\end{array}$ & 1811 & 1832 & 1898 & \\
\hline Bay, A. S. & & 1786 & & 18 & Blackman, John Starr & 1777 & 1808 & 1851 & \\
\hline Bayley \& Douglas & & 1798 & & 18 & Blackman, John Clark & 1808 & 1829 & 1872 & \\
\hline Bayley, S. H. & & 1790 & & 18 & Blakslee, William & 1795 & 1816 & 1879 & \\
\hline Beach, Isaac & & 1788 & & 8 & Blakslee, Ziba & 1768 & 1789 & 1825 & \\
\hline Beach, Miles & 1742 & 1785 & 1828 & 6 & Blondell, Anthony & & 1799 & & 23 \\
\hline Beach \& Sanford & & 1785 & & 6 & Blowers, John & & 1731 & & 11 \\
\hline Beach \& Ward & & 1789 & & 6 & Boelen, Jaboc & & 1702 & & 18 \\
\hline Beal, Caleb & & 1796 & & 11 & Boehme, Charles L. & & 1804 & & 10 \\
\hline Becker, Philip & & 1764 & & 22 & Bogardus, Evardus & & 1698 & & 18 \\
\hline Bedford, J. & & 1800 & & 6 & Bogert, Albert & & 1816 & & 18 \\
\hline Beebe, S. & & 1831 & & 26 & Bogert, N. & & 1810 & & 18 \\
\hline Beecher, Clement & 1778 & 1801 & 1869 & 5 & Bolton, James & & 1790 & & 18 \\
\hline Belknap, Samuel & & 1789 & & 11 & Bontecou, Timothy, & 1693 & 1736 & 1784 & 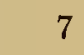 \\
\hline
\end{tabular}




\begin{tabular}{|c|c|c|c|c|c|c|c|c|c|}
\hline Name & Born & Working & Died & age & Name & Born & Working & Died I & Page \\
\hline Bontecou, Timothy, Jr. & 1723 & 1745 & 1789 & 7 & Brigden, Timothy & & 1813 & & 17 \\
\hline Botsford, Gideon B. & 1776 & 1800 & 1866 & 10 & Brigden, $\mathrm{Z}$. & & 1755 & & 15 \\
\hline Boudinot, Elias & & 1749 & & 23 & Bright, Anthony & & 1740 & & 23 \\
\hline Bourdet, Stephen & & 1730 & & 18 & Brinkley, William & & 1804 & & 18 \\
\hline Boutelle, James & & 1783 & & 16 & Broadhurst, Samuel & & 1725 & & 18 \\
\hline Bowder, I. & & 1800 & & 18 & Broek, John & & 1833 & & 18 \\
\hline Bowne, Samuel & & $1 S 05$ & & 18 & Brookhouse, Robert & & 1750 & & 16 \\
\hline Boyce, G. & & 1814 & & 18 & Brower, S. D. & & 1834 & & 17 \\
\hline Boyce, J. & & 1825 & & 18 & Brower, Walter S. & & 1850 & & 17 \\
\hline Boyd \& Hoyt & & 1830 & & 17 & Brown \& Rusher & & 1834 & & 18 \\
\hline Boyd \& Mulford & & 1840 & & 17 & Brown, Ebenezer & & 1793 & & 11 \\
\hline Boyd, Joseph W. & & 1820 & & 17 & Brown, John & & 1796 & & 23 \\
\hline Boyd, William & & 1800 & & 17 & Brown, William & & 1849 & & 17 \\
\hline Boyer, Daniel & & 1748 & & 11 & Brown, Samuel C. & & 1825 & & 18 \\
\hline Braulbury, Theophilas & & 1815 & & 16 & Brown \& Houlton & & 1799 & & 10 \\
\hline Brady, William V & & 1835 & & 18 & Bruff, C. O. & & 1763 & & 18 \\
\hline Bradley, Aner & 1753 & 1780 & 1824 & 7 & Bruff, I. & & 1767 & & 23 \\
\hline Bradley, Luther & & 1798 & & 7 & Bryne, John & & 1790 & & 18 \\
\hline Bradley \& Merriman & & 1817 & & 8 & Bryne, James & & 1792 & & 18 \\
\hline Bradley, Phineas & 1745 & 1779 & 1797 & 7 & Buel, Abel & 1742 & 1770 & 1825 & 7 \\
\hline Bradley, Riehard & 1787 & 1825 & 1867 & 6 & Buel, John & 1744 & 1779 & 1783 & 7 \\
\hline Bradley, Zebel & 1780 & 1806 & 18.59 & 7 & Buel, Simnuel & 1742 & 1777 & 1819 & 7 \\
\hline Bradbury \& Bro. & & $1 \$ 10$ & & 16 & Buel \& Mix & & 1783 & & 7 \\
\hline Brainard, Charles & 1787 & 1809 & 1850 & 6 & Bull, Caleb & $1746^{\circ}$ & 1780 & 1797 & 6 \\
\hline Brasher, Ephraim & & 1786 & & 18 & Bull, Martin & 1744 & 1775 & 1825 & 5 \\
\hline Breed, John & 1752 & 1773 & 1803 & 5 & Bumm \& Shepper & & 1819 & & 23 \\
\hline Brevort, John & & 1742 & & 18 & Bunker, Benjamin & & 1810 & & 26 \\
\hline Brewer, Charles & 1778 & 1800 & 1860 & 7 & Burdock, Nicholas & & 1797 & & 23 \\
\hline Brewer \& Mann & & 1804 & & 7 & Burger, D. I. & & 1725 & & 18 \\
\hline Brewster, Abel & 1775 & 1797 & & 9 & Burger, John & & 1786 & & 18 \\
\hline $\begin{array}{l}\text { Brinton, Gordon \& } \\
\text { Quick }\end{array}$ & & 1780 & & 11 & Burdict, William S. & & 1814 & & 7 \\
\hline Bridge, J. & & 1751 & & 11 & Burnap, Daniel & 1760 & $17 \mathrm{~S} 2$ & 1835 & 5 \\
\hline Brigden, C. & & 1770 & & 11 & Burot, Andrew & & 1824 & & 10 \\
\hline
\end{tabular}




\begin{tabular}{|c|c|c|c|c|c|c|c|c|c|}
\hline Name & Born & Working & Died & Page & Name & Born & Working & \multicolumn{2}{|c|}{ Died Page } \\
\hline Burr, A. G. & & 1815 & & 26 & Cann, John & & 1836 & & 19 \\
\hline Burr, Christopher & & 1810 & & 26 & Cant, Grodtrey & & 1796 & & 19 \\
\hline Burr, C. A. \& Co. & & 1820 & & 26 & Caralin, Pierce & & 1804 & & 19 \\
\hline Burr, Ezekiel & & 1785 & & 26 & Cario, Michael & & 1736 & & 23 \\
\hline Burr, William & & 1792 & & 26 & Carpenter, Joseph & 1747 & 1769 & 1804 & 9 \\
\hline Burr \& Lee & & 1815 & & 26 & Carroll, James & & 1834 & & 17 \\
\hline Burrill, Joseph & & 1823 & & 11 & Carroll, John & & 1825 & & 19 \\
\hline Burrill, S. & & 1733 & & 11 & Carson, David & & 1849 & & 17 \\
\hline Burrill, Theophilus & & 1739 & & 8 & Cary, Lewis & & 1820 & & 11 \\
\hline Burt, Benjamin & 1728 & 1750 & 1803 & 11 & Carmon, John & & 1800 & & 19 \\
\hline Burt, John & 1690 & 1731 & 1745 & 11 & Carman, John & & 1771 & & 23 \\
\hline Burt, Samuel & 1724 & 1746 & 1754 & 11 & Carson, Thomas & & 1808 & & 17 \\
\hline Burt, W. & & 1748 & & 11 & Carson \& Hall & & 1813 & & 17 \\
\hline Bussey, Thomas & & 1799 & & 10 & Case, George & & 1779 & & 5 \\
\hline Bushnell, Phineas & 1741 & 1795 & 1836 & 6 & Casey, Gideon & & 1753 & & 26 \\
\hline Butler, James & 1713 & 1740 & 1776 & 11 & Casey, Samuel & & 1745 & & 25 \\
\hline Butler, John & & 1763 & & 10 & Caston, Françoise & & 1804 & & 19 \\
\hline Butler, N. & & 1803 & & 22 & Champlin, John & 1745 & 1768 & 1800 & 8 \\
\hline Butler \& McCarty & & 1850 & & 23 & Chandless, William & & 1846 & & 19 \\
\hline Cady, Samuel & & 1796 & & 18 & Chapin, Aaron & 1753 & 1825 & 1838 & 6 \\
\hline Cady \& Bachus & & 1796 & & 18 & Charters, James & & 1844 & & 19 \\
\hline Calder \& Co. & & 1830 & & 17 & $\begin{array}{c}\text { Charters, C a n n } \quad \& \\
\text { Dunn }\end{array}$ & & 1850 & & 19 \\
\hline Caldwell, E. & & 1800 & & 18 & Chase, J. D. & & 1820 & & 19 \\
\hline Colwell \& Lawrence & & 1850 & & 17 & Chene, Daniel & & 1786 & & 19 \\
\hline Cameron, Alexander & & 1813 & & 17 & Childs, George K. & & 1837 & & 23 \\
\hline Campbell, R. & & 1824 & & 10 & & & 1816 & & 19 \\
\hline Campbell, R. \& A. & & 1850 & & 10 & Chittenden, Beriah & 1751 & 1787 & 1827 & 7 \\
\hline Campwell, W. & & 1765 & & 23 & Chittenden, Ebenezer & 1726 & 1760 & 1812 & 7 \\
\hline Canavillo, S. & & 1825 & & 18 & Church, Joseph & 1794 & 1820 & 1876 & 6 \\
\hline Candee, Lewis Burton & 1806 & 1825 & 1861 & 10 & Churchill, Jesse & & 1809 & & 11 \\
\hline Canfield, Samuel & & 1780 & & 7 & Churchill \& Treadwell & & 1815 & & 11 \\
\hline Canfield \& Co. & & 1850 & & 10 & Church \& Rogers & & 1825 & & 6 \\
\hline Canfield \& Foot & & 1790 & & 7 & Clapp \& Riker & & 1805 & & is \\
\hline
\end{tabular}




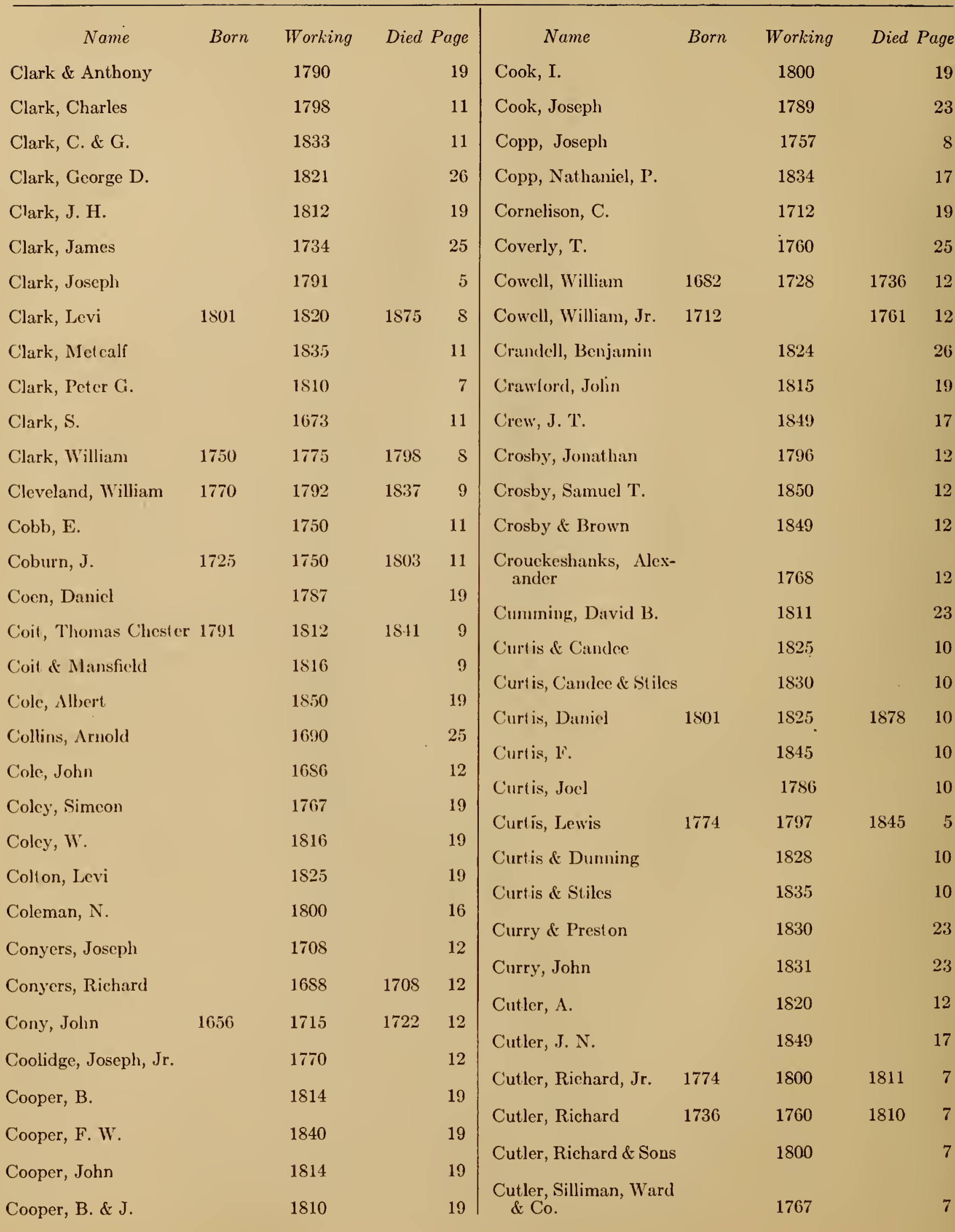




\begin{tabular}{|c|c|c|c|c|c|c|c|c|c|}
\hline Name & Born & Working & Died & age & Name & Born & Working & Died & Page \\
\hline Cutler, William & 1785 & 1800 & 1817 & 7 & Dexter, John & 1735 & 1756 & 1800 & 15 \\
\hline Dagget, Henry & 1741 & 1800 & 1830 & 7 & Dickerson, J. & & 1795 & & 23 \\
\hline Dalley \& Halsey & & 1787 & & 19 & Dickerson, John & & 1797 & & 16 \\
\hline Dane, $\mathrm{T}$. & & 1773 & & 12 & Dixwell, Basil & 1711 & 1732 & 1746 & 12 \\
\hline Davenport, Samuel & 1720 & 1741 & 1793 & 15 & Dixwell, John & 1680 & 1710 & 1725 & 12 \\
\hline Daverne, John & & 1799 & & 10 & Dobbs, Adam & & 1788 & & 19 \\
\hline David, John & & 1755 & 1805 & 23 & Dodge, Cyril & & 1795 & & 26 \\
\hline David, Lewis A. & & 1837 & & 23 & Dodge, Ezra & 1766 & 1798 & 1798 & 8 \\
\hline David, Peter & & 1738 & 1760 & 23 & Dodge, E. & & 1792 & & 19 \\
\hline Davison, Barzillai & 1740 & 1780 & 1828 & 9 & Dodge, Nehemiah & & 1797 & & 26 \\
\hline Davis, E. & & 1775 & & 16 & Doler, Daniel & & 1765 & & 12 \\
\hline Davis, Joshua & & 1796 & & 12 & Donaldson, John W. & & 1823 & & 12 \\
\hline Davis, Palmer \& Co, & & 1841 & & 12 & Doolittle, Amos & 1754 & 1790 & 1832 & 7 \\
\hline Davis, Samuel & & 1801 & & 26 & Doolittle, Enos & & 1781 & & 6 \\
\hline Davis, T. A. & & 1824 & & 12 & Dorsey, Joshua & & 1797 & & 23 \\
\hline Davis, William & & 1823 & & 12 & Douglas, Alexander & & 1792 & & 19 \\
\hline Davis \& Babbitt & & 1820 & & 26 & Douglas, Cantwell & & 1799 & & 10 \\
\hline Davis \& Brown & & 1802 & & 12 & Douglas, J. & & 1792 & & 19 \\
\hline Davis \& Watson & & 1815 & & 12 & Douglas, Robert & 1740 & 1766 & 1776 & 8 \\
\hline Davy, Adam & & 1796 & & 23 & Dowig, George & & 1765 & & 23 \\
\hline Dawes, William & & 1766 & & 12 & Dowig. George & & 1789 & & 10 \\
\hline Dawson, John & & 1767 & & 19 & Drewrey, G. & & 1763 & & 23 \\
\hline Delano, Jabez & 1763 & 1784 & 1848 & 15 & Drowne, Benjamin & & 1800 & & 16 \\
\hline Demmock, John & & 1798 & & 12 & Drowne, Samuel & 1749 & 1770 & 1815 & 16 \\
\hline Denise, J. \& T. & & 1798 & & 19 & Drowne, Shem & 1728 & 1749 & 1774 & 12 \\
\hline Dennis, Ebenezer & & 1778 & & 6 & Drown, T. P. & & 1790 & & 16 \\
\hline Dennis, George, Jr. & & 1778 & & 9 & Dubois, A. & & 1797 & & 23 \\
\hline De Perrizang, Otto & & 1786 & & 19 & Dubois, J. & & 1790 & & 19 \\
\hline De Peyster, William & & 1733 & & 19 & Duche, B. R. & & 1800 & & 19 \\
\hline De Remier, \& Mead & & 1831 & & 18 & Dummer, Jeremiah & 1645 & 1669 & 1718 & 12 \\
\hline De Remier, Peter & & 1769 & & 19 & Dumontet, S. B. & & 1797 & & 23 \\
\hline Deshon, Daniel & 1697 & 1720 & 1781 & 8 & Dumorte, John & & 1796 & & 23 \\
\hline Deverill, J. & & 1787 & & 12 & Dunkerly, Joseph & & 1787 & & 12 \\
\hline
\end{tabular}




\begin{tabular}{|c|c|c|c|c|c|c|c|c|c|}
\hline Name & Born & Working & Died 1 & age & Name & Born & Working & Died & Page \\
\hline Dunlevey, Robert & & 1831 & & 23 & Evans, John & & 1816 & & 19 \\
\hline Dunn, Cary & & 1765 & & 19 & Evans, Robert & & 1798 & & 12 \\
\hline Dupuy, Daniel & 1719 & 1750 & 1805 & 23 & Evertsen, John & & 1806 & & 17 \\
\hline Dupuy, Daniel, Jr. & & 1796 & & 23 & Faber, William & & 1831 & & 23 \\
\hline Dwight, Timothy & 1654 & 1680 & 1692 & 12 & Faber \& Hoover & & 1837 & & 23 \\
\hline Eames, Joshua & & 1700 & & 12 & Fairchild, Joseph & & 1824 & & 7 \\
\hline Easton, James & & 1828 & & 15 & Fairchild, Robert & 1703 & 1770 & 1794 & 5 \\
\hline Easton, Nathaniel & & 1780 & & 15 & Faris, Charles & & 1790 & & 15 \\
\hline Easton \& Stanford & & 1830 & & 15 & Farley, Charles & & 1812 & & 10 \\
\hline Eayas, Thomas S. & & 1798 & & 12 & Farnham, Henry & • & 1800 & & 12 \\
\hline Edmechat, C. & & 1790 & & 19 & Farnliam, Rufus & & 1790 & & 12 \\
\hline Edwards, Andrew & & 1796 & & 12 & Farnham, R. \& H. & & 1800 & & 12 \\
\hline Edwards, Calvin & 1689 & & & 11 & Farringt on, John & & 1833 & & 12 \\
\hline Edwards, John & 1686 & 1710 & 1743 & 12 & $\begin{array}{l}\text { Farrington \& Hunne- } \\
\text { well }\end{array}$ & & 1835 & & 12 \\
\hline Edwards, Joseph & & 1763 & & 12 & Feurt, Peter & & 1727 & 1737 & 12 \\
\hline Edwards, Samuel & & 1762 & & 12 & Fielding, George & & 1731 & & 19 \\
\hline $\begin{array}{l}\text { Edwards, Thomas } \\
\text { Edwards, Thomas }\end{array}$ & 1720 & $\begin{array}{l}1741 \\
1734\end{array}$ & 1755 & 12 & Finch, Hiram & & 1840 & & 17 \\
\hline Elderkin, Alfred & 1759 & 1792 & 1833 & 10 & Fiteh, Allen & & 1808 & & 7 \\
\hline Elderkin, Elishar & 1753 & 1774 & 1822 & 6 & Flagg, Josial & & $\begin{array}{l}1812 \\
1765\end{array}$ & & 12 \\
\hline Elliott, John Aaron & & 1815 & & 9 & liletcher \& Gardner & & 1819 & & 23 \\
\hline Ellison, Peter & & 1792 & & 19 & Fleteher, 'T. & & 1815 & & 23 \\
\hline $\begin{array}{l}\text { Ellsworth, David } \\
\text { Emery, Stcphen }\end{array}$ & 1742 & $\begin{array}{l}1772 \\
1789\end{array}$ & 1821 & $\begin{array}{l}10 \\
12\end{array}$ & Fling, George & & 1749 & & 23 \\
\hline Emery, Thomas K. & & 1800 & & 12 & $\begin{array}{l}\text { Flott, Lewis } \\
\text { Folsom, John }\end{array}$ & & $\begin{array}{l}1817 \\
1781\end{array}$ & & 10 \\
\hline Emery \& Co. & & 1798 & & 12 & Foote, William & & 1796 & 。 & 5 \\
\hline England, William & & 1718 & & 23 & Forbes, A. G. & & 1769 & & 19 \\
\hline Eoff, G. & & 1811 & & 19 & Forbes, B. G. & & 1833 & & 19 \\
\hline Eoff \& Connor & & 1833 & & 19 & Forbes, C. V. G. & & 1816 & & 19 \\
\hline Eoff \& Phyfe & & 1850 & & 19 & Forbes, Garret & & 1816 & & 19 \\
\hline Epps, Ellery & & 1808 & & 12 & Forbes, I. W. & & 1805 & & 19 \\
\hline Erwin, John & & 1817 & & 10 & Forbes, William & & 1835 & & 19 \\
\hline Etting, Benjamin & & 1769 & & 19 & Forbes, William G. & & 1773 & & 19 \\
\hline
\end{tabular}




\section{Name}

Born Working

Forman, Berwin B.

Ford, Samuel

Forest, Alexander

Foster, G.

Foster, John

Foster, Joseph

Foster, Samuel

1676

Fourniquet, L.

Fowler, G.

Francis, Julius C. 1785

Francis, N.

Franciscus, George

Fraser, William

Frobisher, Benjamin C.

Frost \& Mumford

Frothingham, Ebenezer

1756

Fryer, John W.

Fueter, Daniel

Fueter, Daniel C.

Fueter, Lewis

Furt, Peter

Gadley \& Johnson

Gafkins, J.

Gale \& Hayden

Gale, John L.

Gale \& Stickler

Gale, William

Gale, William, Jr.

Gale, W. \& Son

Gale, Wood \& Hughes

Gale \& Willis

Gallup, Christopher 1764

Garrett, P.
Died Page

1813

1797

1802

1838

1795

1789

1797

1796

1825

1807

1810

1817

1735

1834

1810

$1 / 77$

$1814 \quad 12$

1813

1789

1754

1770

1728

1849

1832

1847

1816

1825

1821

1850

1850

1833

1840

1790

1849

1811

12

12

$1702 \quad 12$

12

19

19

1862

7

19

10

24

12

26

17

19

19

20

12

17

26

20

20

20

20

20

20

20
Name

17 Gardner, B.

24 Gardner, Sidney

10 Gardiner, John

16 Garrisson, J.

\section{Gito}

Gay, Nathaniel

Gee, Joseph

Gelston, G. S.

Gelston \& Co.

Gelston \& Treadwell

Born

Working

1734

1753

Gerrish, Timothy

Ghiselin, William

Gibbs, Daniel

Gibbs, John

Gilbert, Samuel

Gilbert, W.

Gilbert \& Cunningham

Gill, Caleb

Gill, Leavitt

Gilley, Peter

Givan, A.

Glidden, Joseph

Goelet, Philip

Gooding, Henry

Goodwin, Allyn

1797

Goodwin, Benjamin

Goodwin, Horace

1787

20

Goodwin, H. \& A.

Goodwin, Ralph

1793

Goodwin \& Dodd

24 Gordon, A.
1828

1810

1760

1825

1680

1788

1833

1836

1836

1797

1788

1819

Died Page

20

12

1776

8

20

12

24

20

20

20

24

24

24

1774

$1813 \quad 16$

1757

24

1716

12

1798

26

1798

1783

1839

20

1789

15

1791

15

1797

24

1849

17

1730

12

1731

20

1833

12

1825

$1869 \quad 6$

1756

12

1808

$1864 \quad 6$

1821

6

1828

$1866 \quad 6$

1812

6

1798 


\begin{tabular}{|c|c|c|c|c|c|c|c|c|c|}
\hline Name & Born & Working & Died & Page & Name & Born & Working & Died I & Page \\
\hline Gordon, A. \& J. & & 1798 & & 20 & Guille, Noah & & 1701 & & 13 \\
\hline Gordon, J. & & 1798 & & 20 & Guirue, Anthony & & 1796 & & 24 \\
\hline Gordon \& Co. & & 1849 & & 12 & Gunn, Enos & 1770 & 1792 & & 9 \\
\hline Gorham, Jabez & & 1814 & & 26 & Gurley, WVilliam & & 1804 & & 9 \\
\hline Gorham, John & & 1841 & & 7 & Gurnee, B. \& S. & & 1833 & & 20 \\
\hline Gorham, Miles & 1757 & 1780 & 1847 & 7 & Haddlock, Henry & & 1850 & & 13 \\
\hline Gorham, Richard & & 1805 & & 7 & Haddock \& Andrews & & 1847 & & 13 \\
\hline Gorham \& Son & & 1841 & & 26 & Hadwen, William & & 1816 & & 26 \\
\hline Gorham \& Thurber & & $18: 1$ & & 26 & 'Harkle, William & & 1766 & & 24 \\
\hline Gorham \& Wcbster & & 1831 & & 26 & Hall, Abijah & & 1813 & & 17 \\
\hline Gough, James & & 1769 & & 20 & Hall, Charles & & 1765 & & 22 \\
\hline Gowen, William & & 1777 & & 15 & Hall, Davidl & & 1765 & & 24 \\
\hline Grant, Thomas & 1731 & 1752 & 1804 & 15 & Hall, Drew & & 1789 & & 20 \\
\hline Grant, William & & 1797 & & 15 & Hall, Green & & 1813 & & 17 \\
\hline Grant, William & 1766 & 1787 & 1809 & 24 & Hall, Joseph & & 1781 & & 17 \\
\hline Gruhan, Daniel & 1764 & 1789 & & 10 & Hall \& Brower & & 1853 & & 17 \\
\hline Gray, John & & 1712 & & 12 & Hall, Brower \& Co. & & 1836 & & 17 \\
\hline Gray, John & 1692 & 1713 & 1720 & 8 & Hall \& Hewson & & 1819 & & 17 \\
\hline Gray, S. & & 1773 & & 13 & $\begin{array}{l}\text { Hall Hewson \& } \\
\text { Brower }\end{array}$ & & 1845 & & 17 \\
\hline Gray, Samuel & 1684 & 1705 & 1713 & S & Hall, Hewson \& & & & & \\
\hline Greculeaf, Davisl & 1737 & 1769 & 1800 & 9 & Merrifield & & 1840 & & 17 \\
\hline Grecnleaf, Davicl, Jr. & 1765 & $178 S$ & 1835 & 6 & Hallam, John & 1752 & 1773 & 1800 & 8 \\
\hline Greenleaf, Joseph & 1778 & 1797 & 1798 & 8 & Halstead, B. & & 1783 & & 24 \\
\hline Greene, Benjamin & 1712 & 1733 & 1748 & 13 & Halstead, Benjamin & & 1764 & & 20 \\
\hline Greene, R. & & 1729 & & 13 & Halstrick, J. & & 1846 & & 13 \\
\hline Greene, William \& Co. & & 1815 & & 26 & Halscy, Jabez & & 1790 & & 20 \\
\hline Griffith, David & & 1789 & & 13 & Hamilton, James & & 1766 & & 11 \\
\hline Griffing, C. & & 1825 & & 20 & Hamlin, IVilliam & & 1793 & & 7 \\
\hline Grigg, W. & & 1765 & & 20 & Hamlin, Cyrus & 1810 & 1831 & 1900 & 10 \\
\hline Grignon, Benjamin & & 1685 & & 13 & Hammerslę, Thomas & & 1756 & & 20 \\
\hline Grignon, Rene & & 1708 & & 9 & Hlam, George & & 1810 & & 16 \\
\hline Griselin, Caesar & & 1700 & & 24 & Hancock, J. & & 1762 & & 13 \\
\hline Griswold, Gilbert & & 1825 & & 7 & Hanks, Benjamin & 1738 & 1777 & 1810 & 10 \\
\hline
\end{tabular}




\begin{tabular}{|c|c|c|c|c|c|c|c|c|c|}
\hline Name & Born & Working & Died & Page & Name & Born & Working & Died & Page \\
\hline Hanners, George & 1696 & 1716 & 1740 & 13 & Heron, Isaac & & 1768 & & 20 \\
\hline Hanners, George, Jr. & 1721 & 1742 & 1760 & 13 & Heurtin, William & & 1731 & & 20 \\
\hline Hansell, Robert & & 1823 & & 13 & Hews, Abram & & 1823 & & 13 \\
\hline Harding, Newill & & 1820 & & 13 & Hewson, John D. & & 1818 & & 17 \\
\hline Harding, N. \& Co. & & 1830 & & 13 & Heyer, W. B. & & 1798 & & 20 \\
\hline Harland, Thomas & 1735 & 1773 & 1807 & 9 & Hilldrup, Thomas & & 1772 & & 6 \\
\hline Harland, Thomas, Jr. & 1781 & 1802 & 1806 & 9 & Hiller, J. & & 1770 & & 16 \\
\hline Harper, T. W. & & 1825 & & 20 & Hinsdale, E. & & 1796 & & 20 \\
\hline Hartin \& Bargi & & 1766 & & 16 & Hitchborn, Samuel & 1752 & 1773 & 1828 & 13 \\
\hline Harris, $\mathrm{H}$. & & 1820 & & 17 & Hitchcock, Eliakim & 1726 & 1776 & 1788 & 7 \\
\hline Harris, Stanwood \& Co & & 1845 & & 13 & Hobart, Joshua & & 1813 & & 8 \\
\hline Harris \& Wilcox & & 1844 & & 17 & Hobbs, Nathan & & 1833 & & 13 \\
\hline Hart, Eliphaz & 1789 & 1810 & 1866 & 8 & Holinghead, William & & 1762 & . & 24 \\
\hline Hart, Judah & 1777 & 1800 & 1824 & 7 & Holton, David & & 1804 & & 10 \\
\hline Hart, J. & & 1825 & & 20 & Holland, Littleton & & 1804 & & 10 \\
\hline Hart \& Bliss & & 1803 & & 7 & Holmes, Israel & 1768 & 1789 & 1802 & 9 \\
\hline Hart \& Brewer & & 1800 & & 7 & Holmes, J. & & 1816 & & 20 \\
\hline Hart \& Wilcox & & 1805 & & 9 & Holyoke, Edward & & 1817 & & 13 \\
\hline Hascy, Alexander & & 1849 & & 17 & Homes, William & 1717 & 1738 & 1783 & 13 \\
\hline Hascy, Nelson & & 1849 & & 17 & Homes, William, Jr. & 1742 & 1763 & 1825 & 13 \\
\hline Haskell, Barnabus & & 1833 & & 13 & Hood \& Tobey & & 1849 & & 17 \\
\hline Hastier, John & & 1726 & 1771 & 20 & Hookey, William & & 1745 & & 25 \\
\hline Hastier, Marquette & & 1771 & & 20 & Hopkins, Jesse & 1766 & 1787 & & 9 \\
\hline Haugh, Samuel & & 1700 & & 13 & Hopkins, Joseph & 1730 & 1766 & 1801 & 9 \\
\hline Hayden \& Gregg & & 1840 & & 20 & Hopkins, Stephen & 1721 & 1771 & 1796 & 9 \\
\hline Hays, Andrew & & 1769 & & 20 & Horn, B. & & 1847 & & 13 \\
\hline Hayes \& Colton & & 1831 & & 17 & Hotchkiss, Hezekiah & & 1751 & 1761 & 8 \\
\hline Heath, John & & 1761 & & 20 & Houlton, John & & 1797 & & 24 \\
\hline Hebbard, H. & & 1847 & & 20 & How, David & & 1790 & & 13 \\
\hline $\begin{array}{l}\text { Hequembourg, Charles } \\
\text { Jr. }\end{array}$ & 1760 & 1809 & 1851 & 8 & Howard, Abraham & & 1810 & & 16 \\
\hline Henchman, Daniel & & 1732 & & 13 & Howard, William & & 1800 & & 13 \\
\hline Hendrickse, A. & & 1698 & & 20 & Howell, James & & 1813 & & 24 \\
\hline Herbert, T. B. & & 1825 & & 20 & Hughes \& Bliss & & 1806 & & 7 \\
\hline
\end{tabular}




\begin{tabular}{|c|c|c|c|c|c|c|c|c|c|}
\hline Name & Born & Working & Died 1 & Page & Name & Born & Working & Died & Page \\
\hline \multicolumn{2}{|l|}{$\begin{array}{l}\text { Hughes, Christopher \& } \\
\text { Co. }\end{array}$} & \multirow[t]{2}{*}{1773} & \multicolumn{2}{|r|}{10} & Johnson, M. W. & & 1815 & & 17 \\
\hline Hughes, Edmond & & & & 7 & Johnson, Samuel & & 1783 & & 20 \\
\hline Hughes \& Francis & & 1807 & & 7 & Johnson \& Goldey & & 1847 & & 17 \\
\hline Hull, John & 1624 & 1659 & 1683 & 13 & Johonnot, William & 1766 & 1787 & 1849 & $\begin{array}{r}7 \\
13\end{array}$ \\
\hline Hull \& Sanderson & & 1652 & & 13 & Jones, J. B. & & 1830 & & 13 \\
\hline Humphreys, Richard & & 1772 & & 24 & Jones, John & & 1810 & & 13 \\
\hline Humphreys, Thomas & & 1814 & & 24 & Jones, Ball \& Co. & & 1835 & & 13 \\
\hline Hunt, Edward & & 1718 & & 24 & Jones, Balt \& Poor & & 1840 & & 13 \\
\hline Huntington, Philip & 1770 & 1791 & 1825 & 9 & Jones \& Ward & & 1815 & & 13 \\
\hline $\begin{array}{l}\text { Huntington, Roswell } \\
\text { Hurd, Benjamin }\end{array}$ & 1763 & 1784 & $17 \$ 1$ & 16 & lieeler, Josepl & 1786 & 1807 & 1824 & 9 \\
\hline Hurd, Jacob & 1702 & 1731 & 1758 & 13 & Keith, T. & & 1790 & & 20 \\
\hline Hurd, Nathaniel & 1729 & 1750 & 1777 & 13 & $\begin{array}{l}\text { Keley, Grael } \\
\text { Kelley, Allen }\end{array}$ & & $1 \$ 10$ & & 26 \\
\hline Hurlbert, Plillip & & 1764 & & 24 & Kolley, E. C. \& J. H. & & 1820 & & 26 \\
\hline $\begin{array}{l}\text { Hurst, Henry } \\
\text { Husband, John }\end{array}$ & 1666 & 1796 & 1718 & 24 & Kendall, C. & & 1792 & & 20 \\
\hline Hutton, Isaac & & 1790 & & 17 & Kenrick, Anwill & & 1775 & & 11 \\
\hline Hutton, Johu & & 1720 & & 20 & $\begin{array}{l}\text { Mancy Cann } \\
\text { Johnson }\end{array}$ & & 1850 & & 20 \\
\hline Hutton, John S. & 1684 & 1705 & 1792 & 24 & KKiersteade, Cornelius & 1674 & 1698 & 1753 & 8 \\
\hline Hyde \& Goodrich & & 1810 & & 17 & Kimberly, W. & & 1792 & & 20 \\
\hline Jackson, Danie] & & 178.3 & & 20 & King, Joseph & & 1770 & & 7 \\
\hline Jackson, James & & 1775 & & 11 & Kingston, John & & 1775 & & 20 \\
\hline Jackson, John & & 1731 & & 20 & Kinney, Thomas & & 1821 & & 9 \\
\hline Jackson, Joseph & & 1804 & & 10 & Kip, Benjamin & & 1702 & & 20 \\
\hline Jacobs, George & & 1802 & & 10 & Kippen, George & 1790 & 1825 & & 7 \\
\hline Jarvis, Munson & 1742 & 1763 & 1825 & 9 & Kirk, Samuel & & 1817 & & 10 \\
\hline Jenckes, John C. & & 1794 & & 26 & Kirk, S. \& Son & & 1830 & & 10 \\
\hline Jenkins, John & & 1796 & & 24 & Kirtland, Joseph P. & 1770 & 1796 & & 7 \\
\hline Jenkins \& Jenkins & & 1850 & & 10 & Kline, B. \& Co. & & 1837 & & 24 \\
\hline Jennings, Jacob & 1729 & 1750 & 1817 & 8 & Kneełand, I. & 1698 & 1729 & 1760 & 13 \\
\hline Jennings, Jacob, Jr. & 1779 & 1800 & & 8 & Krirler, Peter L. & & 1850 & & 24 \\
\hline Jesse, David & & 1698 & & 13 & Kuncher, Jacob & & 1811 & & 24 \\
\hline Johnson, C. & & 1825 & & 17 & Laforme, F. J. & & 1835 & & 13 \\
\hline
\end{tabular}




\begin{tabular}{|c|c|c|c|c|c|c|c|c|c|}
\hline Name & Born & Working & Died & Page & Name & Born & Working & Died 1 & Page \\
\hline Laforme Vincent & $\cdot$ & 1850 & & 13 & Low, John J. \& Co. & & 1828 & & 13 \\
\hline Laforme \& Brother & & 1850 & & 13 & Low, Ball \& Co. & & 1840 & & 13 \\
\hline Lamar, Matthais & & 1797 & & 24 & Lownes, Edward & & 1819 & & 24 \\
\hline Lang, J. & & 1753 & & 16 & Lownes, Joseph & & 1796 & & 24 \\
\hline Lathrop, Rufus & 1731 & 1752 & 1805 & 9 & Lownes, J. \& J. H. & & 1819 & & 24 \\
\hline Lawrie, Robert D. & & 1841 & & 24 & Luscomb, John G. & & 1823 & & 13 \\
\hline Leacock, John & & 1751 & & 24 & Luzerder, Benjamin & & 1796 & & 21 \\
\hline Leach, Charles & & 1789 & & 13 & Lyell, David & & $\mathbf{1 6 9 9}$ & & 21 \\
\hline Leach, Nathaniel & & 1789 & & 13 & Lynde, Thomas & 1748 & 1769 & 1812 & 16 \\
\hline Leach \& Bradley & & 1832 & & 22 & Lyng, John & & 1734 & & 24 \\
\hline Ledell, Joseph & & 1752 & & 20 & Lyng, John B. & & 1761 & & 21 \\
\hline Lee, S. W. & & 1815 & & 26 & Main, David & 1752 & 1773 & 1843 & 9 \\
\hline Legare, Frances & 1636 & 1657 & 1711 & 13 & MacFarlane, John & & 1796 & & 13 \\
\hline Lent, John & & 1787 & & 20 & Maldrid \& Co. & & 1787 & & 21 \\
\hline Le Roux, Bartholomer & & 1739 & & 20 & Mann, Alexander & 1777 & 1804 & & 7 \\
\hline Le Roux, Charles & & 1734 & & 20 & Manning, Daniel & & 1823 & & 13 \\
\hline Le Roux, John & & 1722 & & 20 & $\begin{array}{l}\text { Mansfield, E } 1 \text { is h a } \\
\text { Hyde }\end{array}$ & 1795 & 1816 & & 9 \\
\hline Le Ret, Peter & & 1799 & & 10 & Mansfield, John & & 1654 & & 13 \\
\hline Letelier, John & & 1770 & & 24 & Marble, Simeon & 1777 & 1801 & 1856 & 8 \\
\hline Leverett, Knight & & 1702 & & 13 & Marquand, Frederick & & 1835 & & 21 \\
\hline Lewin, Gabriel & & 1773 & & 10 & Marquand \& Co. & & 1835 & & 21 \\
\hline Lewís, Harvey & & 1819 & & 24 & Marshall, Joseph & & 1819 & & 24 \\
\hline Lewis \& Smith & & 1811 & & 24 & Martin, Peter & & 1756 & & 21 \\
\hline Lewis, Isaac & 1773 & 1809 & 1860 & 6 & & & 1800 & & 21 \\
\hline Lincoln, E. & & 1813 & & 15 & Maverick, Peter R. & & 1786 & & 21 \\
\hline Lincoln \& Reed & & 1835 & & 13 & McClymon, J. C. & & 1805 & & 21 \\
\hline Little, William & & 1775 & & 16 & McFee, John & & 1797 & & 24 \\
\hline Lockwood, F. & & 1845 & & 20 & McHarg, Alexander & & 1849 & & 17 \\
\hline Loud, Asa & 1765 & 1792 & 1823 & 6 & McMullen, John & & 1796 & & 24 \\
\hline Loring, Henry & & 1798 & & 13 & McMullin \& Black & & 1811 & & 24 \\
\hline Loring, Joseph & 1743 & 1788 & & 13 & Mead, Adraince \& Co. & & 1832 & & 18 \\
\hline Low, Francis & 1806 & 1827 & 1855 & 13 & Mecum, George & & 1830 & & 13 \\
\hline Low, John J. & 1800 & 1821 & 1876 & 16 & Mecom, John & & 1770 & & 21 \\
\hline
\end{tabular}




\begin{tabular}{|c|c|c|c|c|c|c|c|c|c|}
\hline Name & Born & Working & Died & Page & Name & Born & Working & \multicolumn{2}{|c|}{ Died Page } \\
\hline Merkler, John H. & & 1788 & & 21 & Moseley, David & 1753 & 1774 & 1812 & 14 \\
\hline Merriman, C. & & 1825 & & 21 & Moses, S. & & 1830 & & 14 \\
\hline Merriman, Marcus & 1762 & 1787 & 1850 & 8 & Moss, Isaac Nichols & 1760 & 1781 & 1840 & 5 \\
\hline Merriman, Marcus, Jr. & & 1826 & & 8 & Mott, W. \& J. & & 1790 & & 21 \\
\hline Merriman, Reuben & 1783 & 1827 & 1860 & 6 & Moulton, Abel & & 1815 & & 10 \\
\hline Merriman, Samucl & 1769 & 1794 & 1805 & 8 & Moulton, Ebenezcr & & 1796 & & 14 \\
\hline Merriman, Silas & 1734 & 1769 & 1805 & 8 & Moulton, Joseph & & 1790 & & 16 \\
\hline Merriman \& Bradley & & 1817 & & $s$ & Moulton, Willian & & 1765 & & 16 \\
\hline Merriman \& Co. & & 1802 & & 8 & Moulinar, John & & 1744 & & 21 \\
\hline Merriman \& Tuttle & & 1802 & & 8 & Nudge \& Co. & & 1848 & & 21 \\
\hline $\begin{array}{l}\text { Merryfick, Thomas } \\
\text { V.Z. }\end{array}$ & & 1840 & & 17 & Mulford, J. H. & & 1835 & & 17 \\
\hline Merrow, Nathan & 1758 & 1783 & 1825 & 5 & Mulford \& Wendell & & 1842 & & 17 \\
\hline Miles, John & & 1769 & & 24 & Munford, H. G. & & $\begin{array}{l}1816 \\
1824\end{array}$ & & 26 \\
\hline $\begin{array}{l}\text { Millner, Thomas } \\
\text { Milne, Edward }\end{array}$ & 1690 & 1711 & 1745 & 13 & Munson, Amos & 1753 & 1776 & 1785 & 8 \\
\hline Milne, F. & & 1800 & & 21 & $\begin{array}{l}\text { Munson, Cornelius } \\
\text { Musgrave. James }\end{array}$ & 1742 & $\begin{array}{l}1763 \\
1797\end{array}$ & & $\begin{array}{r}8 \\
24\end{array}$ \\
\hline Minot, Saınuel & & 1744 & & 14 & Myers, Jolın & & 1797 & & 24 \\
\hline Mitchell, Phincas & & 1812 & & 14 & Myers, Myer & & 1786 & & 21 \\
\hline Mix, Visselıcr & & 1849 & . & 17 & Mygatt, Confort Starr & r 1763 & 1804 & 1823 & 5 \\
\hline Moffat, F. II. & & 1852 & & 17 & Mygatt, David & 1777 & 1804 & 1822 & 5 \\
\hline Moore, E. C. & & 1850 & & 21 & $\begin{array}{l}\text { Mygutt, Eli } \\
\text { Nevill, Richard }\end{array}$ & 1742 & $\begin{array}{l}1793 \\
1764\end{array}$ & 1807 & $\begin{array}{r}5 \\
14\end{array}$ \\
\hline Moore, J. C. & & 1836 & & 21 & Newberry, Edwin C. & & 1828 & & 5 \\
\hline Moore, Robert & & 1775 & & 11 & Newhall, Dudley & & 1730 & & 16 \\
\hline Moore \& Brown & & 1833 & & 21 & Newkirk, J. & & 1710 & & 21 \\
\hline Morris, John & & 1797 & & 21 & Nichols, Bassett & & 1815 & & 26 \\
\hline Morris, Sylvester & & 1759 & & 21 & Nichols, W, S. & 1785 & 1808 & 1871 & 25 \\
\hline Morse, David & & 1798 & & 14 & Nickerson, Baty & & 1825 & & 15 \\
\hline Morse, Hazen & & 1813 & & 14 & Norcross, Nehemiah & & 1796 & & 14 \\
\hline Morse, Moses & & 1825 & & 14 & Norton, Andrew & 1765 & 1786 & 1838 & 5 \\
\hline Morse, Nathaniel & & 1748 & & 14 & Norton, Benjamin & & 1810 & & 14 \\
\hline Morse, Stephen & 1743 & 1796 & & 14 & Norton, Thomas & 1773 & 1796 & 1834 & 5 \\
\hline
\end{tabular}




\begin{tabular}{|c|c|c|c|c|c|c|c|c|c|}
\hline Name & Born & Working & Died & Page & Name & Born & Working & Died & Page \\
\hline Norton, Samuel & & 1795 & & 15 & Pettit, Thomas & & 1792 & & 21 \\
\hline Noyes, John & 1674 & 1695 & 1749 & 14 & Phelps, Jedediah & & 1781 & & 26 \\
\hline Noyes, Samuel & 1747 & 1768 & 1781 & 9 & Phil]ips, Samuel & & 1721 & & 16 \\
\hline Oakes, Frederick & & 1825 & & 6 & Pierce, John & & 1810 & & 14 \\
\hline Oakes \& Spencer & & 1814 & & 6 & Pierpont, Benjamin & & 1760 & & 14 \\
\hline Olgier, John & & 1799 & & 10 & Pinchin, William & & 1784 & & 24 \\
\hline Olivier, Peter & & 1797 & & 24 & Pinto, Joseph & & 1758 & & 21 \\
\hline Olmstead, Nathaniel & 1785 & 1808 & 1860 & 5 & Pitkin, Henry & & 1834 & & 5 \\
\hline Onclebag, Gerrit & & 1690 & & 21 & Pitkin, James 1. & & 1834 & & 5 \\
\hline Osgood, J. & & 1817 & & 16 & Pitkin, John O. & 1803 & 1826 & 1891 & 5 \\
\hline Otis, Jonathan & 1723 & 1748 & 1791 & 25 & Pitkin, Walter & 1808 & 1826 & 1885 & 5 \\
\hline Overin, Richard & & 1701 & & 21 & Pitman, John K. & & 1805 & & 26 \\
\hline Paddy, Samuel & & 1669 & & 14 & Pitman, Saunders & 1732 & 1753 & 1804 & 26 \\
\hline Parisien, Otto & & 1762 & & 21 & Pitman, William R. & & 1835 & & 15 \\
\hline Parisien, Otto \& Son & & 1789 & & 21 & Pitman \& Dodge & & 1790 & & 26 \\
\hline Parker, Daniel & & 1763 & & 14 & Pitman \& Dorrance & & 1795 & & 26 \\
\hline Parker, George & & 1804 & & 11 & Pitt, Richard & & 1741 & & 24 \\
\hline Parker, Isaac & & 1780 & & 15 & Platt, George W. & & 1825 & & 21 \\
\hline Parkman, J. & & 1746 & & 14 & Platt \& Brother & & 1796 & & 21 \\
\hline Parmele, James & 1763 & 1784 & 1828 & 5 & Poineignon, Francis & & 1796 & & 24 \\
\hline Parmele, Samuel & 1737 & 1758 & 1803 & 6 & Poissonier, F. & & 1797 & & 24 \\
\hline Paton, A. & & 1850 & & 14 & Polgrain, Quom & & 1797 & & 24 \\
\hline Pattit, Thomas & & 1796 & & 21 & Polhamus, J. & & 1839 & & 21 \\
\hline Peabody, John & & 1779 & & 5 & Pollard, William & 1690 & 1711 & & 14 \\
\hline Pear, Edward & & 1833 & & 14 & Pons, Thomas & & 1783 & & 14 \\
\hline Pear \& Becall & & 1850 & & 14 & Poor", Nathaniel C. & 1808 & 1829 & 1895 & 14 \\
\hline Pearce, Samuel & & 1783 & & 21 & Portram, Abraham & & 1727 & & 21 \\
\hline Pearson, John & & 1796 & & 21 & Post, Samuel & 1736 & 1783 & & 8 \\
\hline Peck, Timothy & 1765 & 1791 & 1818 & 7 & Poupard, James & & 1772 & & 24 \\
\hline Pelletrau, E. & & 1750 & & 21 & Potwine, John & 1698 & 1737 & 1792 & 14 \\
\hline Perkins, Houghton & & 1762 & 1777 & 14 & Potwine \& Whiting & & 1738 & & 6 \\
\hline Perkins, Jacob & & 1787 & & 16 & Pratt, Nathan & 1772 & 1792 & 1842 & 5 \\
\hline Perraux, Peter & & 1797 & & 24 & Pratt, Nathan. Jr. & 1802 & 1823 & & 5 \\
\hline
\end{tabular}




\begin{tabular}{|c|c|c|c|c|c|c|c|c|c|}
\hline Name & Born & Working & Died & Page & Name & Born & Working & Died & Page \\
\hline Pratt, Phineas & 1747 & 1772 & 1813 & 9 & Ridgeway, James & & 1789 & & 14 \\
\hline Pratt, Seth & 1741 & 1762 & 1802 & 7 & Ridgeway, John & & 1807 & & 14 \\
\hline Price, Benjamin & & 1767 & & 24 & Riker, $\mathrm{P}$. & & 1801 & & 21 \\
\hline Price, John & & 1764 & & 22 & Riker \& Alexander & & 1798 & & 21 \\
\hline Prince, Job & & 1701 & 1703 & 7 & Ritter, Michael & & 1786 & & 21 \\
\hline Putnam, Edward & & 1810 & & 16 & Roath, Roswell W. & 1805 & 1826 & & 9 \\
\hline Putnam \& Low & & 1822 & & 14 & Robert, Christopher & & 1731 & & 21 \\
\hline Quintard, Peter & 1699 & 1731 & 1762 & 21 & Roberts, Frederick & & 1770 & & 14 \\
\hline Rasch, Anthony & & 1815 & & 24 & Robcrts, Michael & & 1786 & & 21 \\
\hline Rasch \& Willig & & 1819 & & 24 & Robinson \& Harwood & . & 1819 & & 25 \\
\hline Recd, Isaac & 1746 & 1776 & & 9 & Rockwell, E. & & 1825 & & 21 \\
\hline Reed, Osman & & 1843 & & 24 & Rockwell, Thomas & & 1790 & & 9 \\
\hline Reeder, Abner & & 1797 & & 24 & Roe, W. & & 1805 & & 21 \\
\hline Recves, Stephen & & 1767 & & 16 & Rogers, A. & & 1840 & & 14 \\
\hline Revere, Edward & 1767 & $178 \mathrm{~S}$ & 1845 & 14 & Rogers, Danicl & & 1750 & & 26 \\
\hline Revere, J. II. & & 1798 & & 14 & Rogers, Joseph & & 1760 & 1825 & 26 \\
\hline Reverc, Piıul & 1702 & 1729 & 1754 & 14 & Rogers, William & 1801 & 1823 & 1873 & 6 \\
\hline Revere, Paul & $17: 35$ & 1760 & 1818 & 14 & Rogers \& Wendt & & 1850 & & 14 \\
\hline Revere, Paul & 1760 & 1780 & 1813 & 14 & Rominire, Jolnn & & 1770 & & 21 \\
\hline Revere, Thomas & 1765 & 1786 & 1817 & 14 & Roosevelt, N. & & 1735 & & 21 \\
\hline Revere \& Son & & 1796 & & 14 & Roshore, John & & 1796 & & 21 \\
\hline Rice, Joseph 'T. & & 1813 & & 17 & Roshore \& Prime & & 1825 & & 21 \\
\hline Rich, Joscph & & 1790 & & 24 & Russell, John H. & & 1796 & & 21 \\
\hline Rich, Obadiah & & 1835 & & 14 & Russell, Jonathan & 1770 & 1804 & & 5 \\
\hline Richardson, Francis & & 1738 & & 24 & Rouse, Michael & 1687 & 1711 & & 14 \\
\hline Richardson, Joseph & & 1736 & & 24 & Rouse, Villiam & 1639 & 1660 & 1704 & 14 \\
\hline Richardson, J. & & 1796 & & 24 & Royalston, John & & 1770 & & 14 \\
\hline Richardson, Thomas & & 1769 & & 21 & Sackett \& Williard & & 1815 & & 26 \\
\hline Richard, Samuel & & 1797 & & 24 & Sadd, Hervey & 1776 & 1798 & 1840 & 6 \\
\hline Richards, S. & & 1828 & & 21 & Sadtler, Phillip & & 1824 & & 11 \\
\hline Richards \& Williamson & & 1797 & & 24 & Saint, Martin Anthony & & 1796 & & 25 \\
\hline Richmond, G. \& A. & & 1815 & & 26 & Sanderson, Robert & & 1650 & 1693 & 14 \\
\hline Ridout, George & & 1748 & & 21 & Sanderson, Robert, Jr. & 1652 & 1673 & 1714 & 16 \\
\hline
\end{tabular}




\begin{tabular}{|c|c|c|c|c|c|c|c|c|}
\hline Name & Born & Working & Died & Page & Name & Born & Working & Died I \\
\hline Sanderson, W. & & 1800 & & 21 & Simmons, Andrew & & $\mathbf{1 7 9 6}$ & \\
\hline Sanford, F. S. & . & 1830 & & 15 & Simmons, Anthony & & 1797 & \\
\hline Sanford, Isaac & & 1783 & & 6 & Simpkins, Thomas B. & 1728 & 1749 & 1804 \\
\hline Sanborn, A. & . & 1850 & & 15 & Simpkins, William & 1704 & 1726 & 1780 \\
\hline Sargeant, Jacob & 1761 & 1785 & 1843 & 6 & Simpson \& Beckel & & 1849 & \\
\hline Sardo, Michael & & 1817 & & 11 & Singleton \& Young & & 1800 & \\
\hline Sargeant, Ensign & & 1823 & & 14 & Skerret, Joseph & & 1797 & \\
\hline Savage, Thomas & & 1695 & & 14 & Skerry, G. W. & & 1837 & \\
\hline Sawin; Silas & & 1823 & & 14 & Skinner, Abraham & & 1756 & \\
\hline Sayre, Joel & 1778 & 1798 & 1818 & 21 & Skinner, Elizer & & 1826 & 1858 \\
\hline Schaats, B. & & 1708 & & 21 & Skinner, Thomas & & 1750 & \\
\hline Schenck, Gared & & 1792 & & 21 & Slydell, Joshua & & 1765 & \\
\hline Schenck, John & ; & 1796 & & 21 & Smith, Ebenezer & & 1775 & \\
\hline Scofield, S. & & 1815 & & 17 & Smith, I. & & 1742 & \\
\hline Seal, William & & 1819 & & 25 & Smith, I. & & 1842 & \\
\hline Sell, J. & & 1800 & & 21 & Smith, Joseph & & 1789 & \\
\hline Sexnine, Simon & & 1722 & & 21 & Smith, James & & 1797 & \\
\hline Sharp, W. \& G. & & 1850 & & 25 & Smith, William & & 1770 & \\
\hline Shaw \& Dunlevey & & 1836 & & 25 & Smith, John \& Thoma & & 1817 & \\
\hline Sheilds, Thomas & & 1765 & & 25 & Sougue, M. & & 1800 & \\
\hline Shepherd, Robert & & 1805 & & 17 & Soumane, Samuel & & 1765 & \\
\hline Shepherd \& Boyd & & 1810 & & 17 & Soumine, Simon & & $171 \dot{9}$ & \\
\hline Shepper, John D. & & 1819 & & 25 & - Spencer, George & 1787 & 1801 & 1878 \\
\hline Shethar, Samuel & & 1810 & & 8 & Spencer, James & & 1793 & \\
\hline Shethar \& Gorham & & 1806 & & 8 & Squire \& Bro. & · & 1846 & \\
\hline Shethur \& Thompson & & 1801 & & 6 & Staats, Bartholomew & & 1784 & \\
\hline Shipman, Nathaniel & 1764 & 1790 & 1853 & 9 & Stacy, P. & & 1819 & \\
\hline Shoemaker, Joseph & & 1797 & & 25 & Staniford, John & & 1785 & \\
\hline Shreve, Benjamin & 1813 & 1834 & 1896 & 14 & Stall, Joseph & & 1804 & \\
\hline Sibley, Clark & 1778 & 1799 & 1808 & 8 & Stanton, Daniel & 1755 & 1773 & 1781 \\
\hline Sibley \& Marble & & 1801 & & 8 & Stanton, Enoch & 1745 & 1765 & 1781 \\
\hline Silliman, Hezekiah & 1738 & 1767 & & 8 & Stanton, Zebulon & 1753 & 1773 & 1828 \\
\hline Simes, William & & 1800 & & 16 & Stanwood, Henry B. & 1818 & 1839 & 1869. \\
\hline
\end{tabular}




\begin{tabular}{|c|c|c|c|c|c|c|c|c|c|}
\hline Name & Born & Working & Died & Page & Name & Born & Working & Died & Page \\
\hline Stanwood, James D. & & 1846 & & 14 & Terry, Geer & 1775 & 1814 & 1838 & 5 \\
\hline Stanwood \& Halstrick & & 1850 & & 14 & Thaxter, Joseph & & 1850 & & 15 \\
\hline Staples, John J. Jr. & & 1788 & & 22 & Thibault \& Co. & & 1797 & & 25 \\
\hline Stebbins \& Co. & & 1825 & & 22 & Thomas, W. & & 1769 & & 22 \\
\hline Stebbins \& Howe & & 1815 & & 22 & Thompson, Isaae & & 1798 & & 6 \\
\hline Stephens, George & & 1790 & & 22 & Thomson, James & & 1839 & & 22 \\
\hline Steven, George & & 1719 & & 22 & Thomson, Peter & & 1817 & & 15 \\
\hline Stevens \& Lakeman & & 1825 & & 16 & Thomson, IV. & & 1830 & & 22 \\
\hline Stewart C. & & 1840 & & 22 & Titcomb, Francis & & 1813 & & 16 \\
\hline Stickney, Jonathan & & 1796 & & 16 & Tiley, James & 1740 & 1765 & 1792 & 6 \\
\hline Stiles, Benjamin & & 1835 & & 10 & Tingley, Samuel & & 1796 & & 25 \\
\hline Stilman, William & 1767 & $17 s S$ & $18.5 \mathrm{~S}$ & 25 & Tingley, Samuel & & 1767 & & 22 \\
\hline Stodcler \& Frobisher & & 1817 & & 14 & Tompkins, Edmond & 1757 & 1779 & & 9 \\
\hline Stone, Adlum & & 1804 & & 11 & Towzell, John & & 1756 & & 16 \\
\hline Stone of Osborn & & 1796 & & 22 & Tracy, Erastus & 1768 & 1790 & 1795 & 9 \\
\hline Stollenwerk id Co. & & 1800 & & 22 & Trucy, Gordon & 1767 & 1787 & 1792 & 9 \\
\hline Storrs \& Cooley & & 1832 & & 22 & Trott, Jonathain & 1730 & 1770 & 1815 & 15 \\
\hline Stoutenburgh, $T$. & & 1731 & & 22 & Trott, Jonathan, Jr. & 1771 & 1800 & 1813 & 8 \\
\hline Stuart, H. & & 1800 & & 22 & Trott, Joln P. & 1769 & 1810 & 1852 & 8 \\
\hline Sutherland, Goorge & & 1810 & & 14 & Trott \& Brooks & & 1798 & & $\varepsilon$ \\
\hline Sutton, Robert & & 1820 & & 8 & Trott \& Cleveland & & 1792 & & 8 \\
\hline Swan, Caleb & 1754 & 1775 & 1816 & 14 & Truax, Henry R. & & 1815 & & 17 \\
\hline Swan, Robert & & $17+2$ & & 14 & Turner, James & & 1744 & 1759 & 15 \\
\hline Swan, William & 1715 & 1736 & 1774 & 16 & Tutlill, Christopher & & 1730 & & 25 \\
\hline Symmes, John & & 1766 & & 14 & Tuttle, Bethuel & 1779 & 1802 & 1813 & 8 \\
\hline Syng, Phillip & 1676 & 1697 & 1739 & 25 & Tuttle, William & 1800 & 1821 & 1849 & 8 \\
\hline Syng, Phillip & 1703 & 1724 & 1789 & 25 & Tyler, Andrew & 1692 & 1713 & 1741 & 15 \\
\hline Tanner, Jolın & & 1740 & & 26 & Tyler, David & 1760 & 1781 & 1804 & 15 \\
\hline Targec, Jolın & & 1796 & & 22 & Tyler, George & 1740 & 1761 & & 15 \\
\hline Targee, Peter & & 1798 & & 22 & Ufford \& Burdick & & 1814 & & 8 \\
\hline Taylor, William & & 1772 & & 25 & Underhill, A. & & 1780 & & 22 \\
\hline Taylor \& Lowrie & & 1841 & & 25 & Underhill, Thomas & & 1789 & & 22 \\
\hline Ten Eyck, C. & & 1716 & & 22 & Underhill \& Vernon & & 1786 & & 22 \\
\hline
\end{tabular}


Name

Born

Working

Van Bergen, John

Van Beuren, $\mathbf{P}$.

Van Beuren, William

Vanderhan, J.

Vanderspiegel, J.

Van Dyke, Peter

Van Dyke, Richard

Van Voorhis, Daniel

Van Voorhis, Daniel

Van Voorhis \& Cooley

Van Voorhis \& Son

Veazie, Joseph

Vergereau, P.

Vernon, John

Vernon, N.

Vernon, Samuel

Vernon, J. \& Co.

Vilant, William

Vinton, David

Vincent, Richard

Walker, George

Walker, William

Walworth, Daniel

1760

Ward, Ambrose

Ward, Billious

1735 .

1729

Ward, John

Ward, John

Ward, James

1768

Ward, Richard

Ward, Samuel

Ward, Timothy

Ward, William

1736

Ward, William
1813

1790

1797

1740

Died Page

18

22

22

25

1702

1708

1750

1789

1782

1786

1798

1815

1721

1789

1800

1784

1798

1725

1792

1799

1796

1796

1783

1767

1750

1811

1805

1789

1815

1824

1763

1757

1726

\section{2}

22

22

22

25

22

22

26

1737

1830

1808

1777

1777

1856

1768

1826

1761
Name

Born

Working

Died Page

Ward \& Bartholomew $\&$ Brainard

Ward \& Bartholomew

Ward \& Cox

Ward \& Hughes

22

22

26

26

22

25

26

11

25

25

25

Ward \& Rich

Wardin, Daniel

Warner, Andrew E.

Warner, Andrew E., Jr.

Warner, A. E. \& T. H.

Warner, Joseph

Warner, Samuel

Warner, Thomas $\mathrm{H}$.

Waters, S.

Waterman, George

Watts, J. W.

Webb, Barnebus

Webb, George W.

Webb, James

Webster, H. L.

Wedge, $\mathbf{S}$.

Weeden, Peleg

Welles, Andrew

Welles, A. \& G.

Welles, G. I.

Wells, William

Wells \& Co.

(

Wendell \& Roberts

15

Wenman, $B$.

15

West, B.
1809

1804

1811

1805

1833

1811

1811

1837

1805

1811

1797

1814

1790

1849

1844

1762

1850

1817

1831

1804

1803

1783

1805

1860

1804

1813

1766

1787

1800

1850

1789

1770

1830

1790

1815

\section{6}

6

25

7

15

5

11

11

11

25

25

11

25

18

25

15

11

11

26

11

26

6

15

15

6

15

18

22

15 


\begin{tabular}{|c|c|c|c|c|c|c|c|c|c|}
\hline Name & Born & Working & Died & Page & Name & Born & Working & Died I & Page \\
\hline Whartenby, John & & 1831 & & 25 & Wilson, Robert & & 1819 & & 22 \\
\hline Whartenby, Thomas & & 1811 & & 25 & Wilson, William & & 1850 & & 25 \\
\hline Whartenby, T., \& Co. & & 1850 & & 25 & Wilson, R. \& W. & & 1831 & & 25 \\
\hline Whitaker \& Green & & 1825 & & 26 & Wiltberger, Christain & & 1793 & & 25 \\
\hline White, Amos & 1745 & 1766 & 1825 & 5 & Windover, J. & & 1694 & & 22 \\
\hline White, Peregrine & 1747 & 1774 & 1834 & 10 & Winslow, Edward & 1669 & 1702 & 1753 & 15 \\
\hline White, Peter & 1718 & 1738 & 1803 & 9 & Wishart, $\mathrm{H}$. & & 1789 & & 22 \\
\hline Whiton, E. & & $1 \$ ? 26$ & & 15 & Wood, J. E. & & 1845 & & 22 \\
\hline Whiting, Charles & 1725 & 1750 & 1765 & 9 & Wood \& Hughes & & 1845 & & 22 \\
\hline Whitlock, Thomas & & 1796 & & 22 & Woods, Freeman & & 1790 & & 22 \\
\hline Wilcox, Alvan & 1783 & 1805 & 1865 & S & Woodward \& Grosjean & & 1847 & & 15 \\
\hline Wilcox. Cyprian & 1795 & 1827 & 1875 & S & Woolward, Antipas & 1763 & 1791 & & 7 \\
\hline Williams, Deorlitt & & 1776 & 1781 & 6 & Woodward, Eli & & 1812 & & 15 \\
\hline Williams, Sammel & & 1797 & & 25 & Wool, J. W. & & 1791 & & 22 \\
\hline Willi:ms, Steplın & & 1799 & & 26 & Wright, Alexander & & 1775 & & 11 \\
\hline Willig, George & & $1 \$ 19$ & & 25 & Wriggins, Thomus & & 1840 & & 25 \\
\hline Williamson, Samuel & & 1796 & & 25 & Wyatt, Joseph & & 1797 & & 25 \\
\hline Willis, Stilman & & $1 S 23$ & & 15 & Wyncoope, I3. & & 1698 & & 22 \\
\hline Wilmot, Samuel & 1777 & $1 S 0 S$ & 1846 & 8 & Wyncoope, C. & & 1727 & & 22 \\
\hline Wijnot \& Stillman & & $1 S 00$ & & 8 & Yeomans, Elijals & 1738 & 1794 & 1794 & 6 \\
\hline Wilson, Albert & & 1834 & & 18 & Yettons, Randell & & 1739 & & 25 \\
\hline Wilson, George & & 18.50 & & 25 & Young, Ebenezer & & $177 \mathrm{~s}$ & & 6 \\
\hline Wilson, $\mathrm{H}$. & & 1812 & & 25 & Young, Levi & & 1827 & & 5 \\
\hline Wilson, Robert & & 180.5 & & 25 & Young, William & & 1761 & & 25 \\
\hline
\end{tabular}




\section{.}

. 


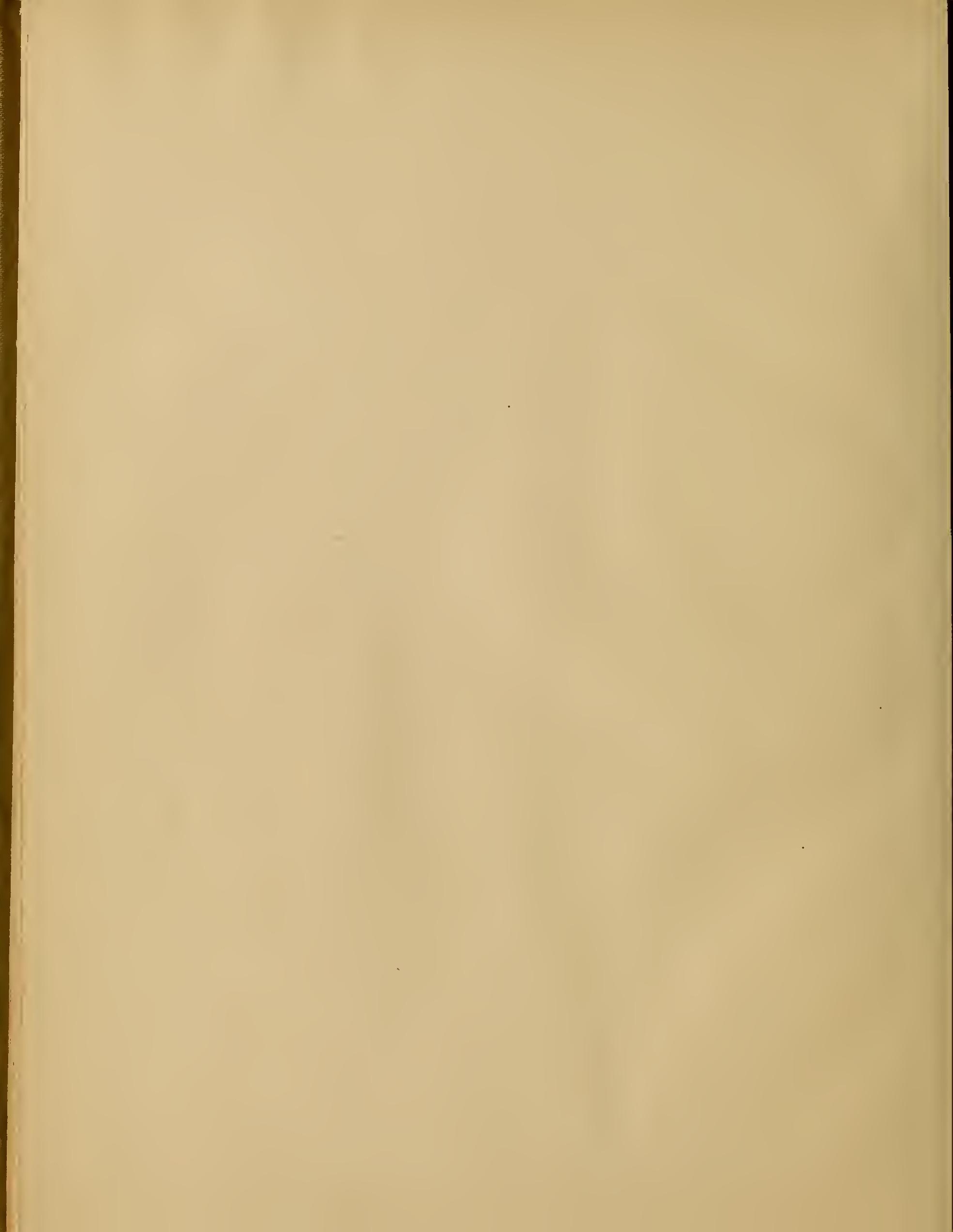


. 

SMITHSONIAN INSTITUTION LIBRARIES

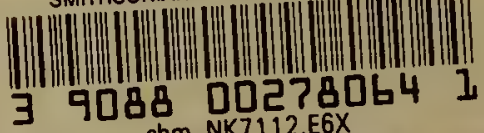
Makers of early American silver. 